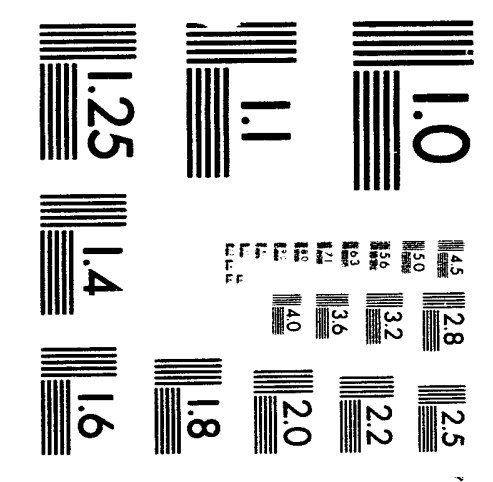


PNL-SA-22813

\title{
NEUTRON INTERACTIONS AND ATDMIC RECOIL SPECTRA
}

L. R. Greenwood

August 1993

\author{
Presented at the \\ International Summer School on \\ Fundamentals of Radiation Damage \\ August 1-12, 1993 \\ Urbana, Illinois
}

Work supported by

the U.S. Department of Energy

under Contract DE-ACO6-76RLO 1830

Pacific Northwest Laboratory

Richland, Washington 99352

\section{DISCLAIMER}

This report was prepared as an account of work sponsored by an agency of the United States Government. Neither the United States Government nor any agency thereof, nor any of their employees, makes any warranty, express or implied, or assumes any legal liability or responsibility for the accuracy, completeness, or usefulness of any information, apparatus, product, or process disclosed, or represents that its use would not infringe privately owned rights. Reference herein to any specific commercial product, process, or service by trade name, trademark, manufacturer, or otherwise does not necessarily constitute or imply its endorsement, recommendation, or favoring by the United States Government or any agency thereof. The views and opinions of authors expressed herein do not necessarily state or reflect those of the United States Government or any agency thereof. 


\section{Neutron Interactions and Atomic Recoil Spectra}

L. R. Greenwood

Battelle, Pacific Northwest Laboratories

\section{Introduction}

This chapter presents a discussion of neutron interactions with materials that lead to activation, transmutation, and atomic displacements. The emphasis will be on current applications including neutron irradiation facilities, neutron dosimetry techniques, and computer codes for spectral adjustment and radiation damage calculations.

The effects of neutron irradiations on materials depend on the neutron spectra and the length of irradiation. Materials effects have been measured in many different types of facilities, including fission reactors and accelerator-based neutron sources. If we want to compare materials effects produced by different types of facilities, then we must consider an exposure parameter that takes into account the differences in the neutron spectrum. For example, we cannot compare an irradiation at a $14 \mathrm{MeV}$ neutron source with one at a fission reactor on the basis of neutron exposure alone since $14 \mathrm{MeV}$ neutrons produce significantly more damage per neutron than lower energy neutrons; furthermore, the nuclear transmutation may be quite different for these two facilities. A widely used exposure parameter that accounts for some of these spectral differences is displacements per atom (dpa), which is a calculated representation of the number of primary and secondary atoms that are displaced from their lattice sites as a result of neutron bombardment. Dpa incorporates, to a first approximation at least, the neutron energy dependent response of the material under irradiation. This is illustrate in Figure 1, which shows that some materials effects measured for 316 stainless steel correlate quite well on the basis of dpa for three radically different spectra including $14 \mathrm{MeV}$, a fission reactor, and a $B e(d, n)$ neutron source.[1] The rest of this chapter will describe the techniques and measurements that are used to perform neutron spectral measurements and subsequent radiation damage calculations to determine neutron irradiation exposure parameters that can be 
used to correlate matciials effects between widely different types of facilities and to predict materials effects in other facilities, such as fusion power reactors.

\section{Neutron Sources and Spectra}

$$
\begin{aligned}
& 35024 \\
& 671
\end{aligned}
$$

Several terms are used to define neutron exposure. Flux is defined as the number of neutrons passing through a unit area regardless of their direction of travel; flux units are thus neutrons $/ \mathrm{cm}^{2}-\mathrm{s}$. Fluence is simply time integrated flux with units of neutrons $/ \mathrm{cm}^{2}$. The neutron energy spectrum describes the energy dependence of the reutron flux. Neutron energies of interest typically run from below room temperature at $0.0253 \mathrm{eV}$ (referred to as thermal neutrons) up to about $20 \mathrm{MeV}$. Neutrons have about the same mass as a proton; however, neutrons differ in that they have no electrical charge. Hence, unlike protons, neutrons can strongly interact with atoms at very low energies. The most common types of reactions that we are concerned with include scattering (like billiard balls), inelastic scattering (where the target nucleus is left in an excited state), and neutron capture (where the atomic weight of the target is increased by one mass unit). Following neutron capture, the nucleus may undergo gamma emission, fission, charged particle reactions (where a proton or alpha particle is emitted) or multiple neutron emission. These latter reactions can produce transmutation that is defined as the change from one element to another. It is easy to predict such effects using the Table of the Isotopes as a guide to determine the reaction products for each reaction as well as the subsequent radioactive decay products. Frequently, the transmuted element may be produced as a radioactive isotope; this is referred to as neutron activation.

Neutrons are produced either from nuclear fission of heavy elements, such as uranium, or by charged particle reactions induced by accelerators. In the fission process, two or more neutrons are produced for every fission. The fission neutron energy spectrum can be described as a maxwellian with a nuclear temperature of about $1.5 \mathrm{MeV}$, as shown in Figure 2. Two basic types of fission reactors have been developed: - fast 
reactors and mixed-spectrum reactors. These differ in the type of coolant and moderator that is used to control the reactor.

Fast reactors are cooled by liquid sodium and thus have no significant moderation. The term "fast reactor" or "fast flux" refers to the relative velocity (energy) of the neutrons. Hence, the neutron spectrum in core is only slightly softer than the basic fission spectrum. An example of this is shown in Figure 3 for the Fast Flux Test Facility (FFTF) at Westinghouse Hanford (WA).[2,3] Such facilities produce high fluxes of fast neutrons without any measurable thermal or epithermal neutron fluxes.

The more common types of fission reactors including most commercial power reactors as well as most research reactors, are water cooled. These reactors are referred to as mixed spectrum reactors since the hydrogen in the water coolant provides substantial moderation of the neutron energy spectrum, leading to roughly equal fluxes of fast and thermal neutrons. This is also illustrated in Figure 3 for the High Flux Isotopes Reactor (HFIR) at Oak Ridge National Laboratory (TN).[4] The term "moderation" refers to the slowing down of neutrons due to scattering, principally with the large amount of hydrogen in the water coolant. Since the nucleus of a hydrogen atom is a proton with equal mass as that of a neutron, the average neutronproton scattering results in the neutron losing about half of its incoming energy. This energy is carried away by the recoiling proton and is lost by heating the water coolant. The hydrogen scattering cross section is also quite large (about 20 barns) so that hydrogen is thus the most effective neutron moderator we know of.

The HFIR spectrum shown in Figure 3 is characteristic of mixed spectrum reactors. The spectrum is plotted as flux per unit lethargy, which simply means neutron energy times neutron flux. There are several different ways to plot a neutron spectrum, as illustrated in Figure 4. A differential plot shows the neutron flux at a point in energy and has units of $n / \mathrm{cm}^{2}-\mathrm{s}-\mathrm{MeV}$. This is somewhat misleading since very low energy neutrons appear to be quite high due to the very small energies in the denominator. 
A group plot shows the number of neutrons in a given energy bin. This representation may also be misleading unless the energy bins have equal width. Maintaining constant width energy bins is not practical since no detail would be provided at the lower neutron energies. The lethargy plot solves this problem by having the bins change logrithmically. For example, each bin could be $10 \%$ wider than the preceding bin. This also has a practical limitation since the higher energy bins become unacceptably large. Hence, my favorite way to plot neutrons is flux per unit lethargy. This is defined as flux/d(InE) which is equal to $E x$ flux. The reason I favor this type of plot is that it gives the best visual picture of relative neutron energies. Units for a flux/lethargy plot are $\mathrm{n} / \mathrm{cm}^{2}-\mathrm{s}$, the same as group plots. The difference is that the group value is equal to the width of the energy bin (dE) times the differential flux whereas the lethargy value is equal to the mid energy of the bin (E) times the differential flux.

Looking at the lethargy plots in Figures 3 and 4 for the HFIR mixed spectrum reactor, we note that the highest part of the neutron spectrum ( $>1 \mathrm{MeV}$ ) looks like a fission spectrum, the intermediate energy spectrum $(0.5 \mathrm{eV}<\mathrm{E}<1 \mathrm{MeV})$ is flat, and the lowest energies ( $<0.5 \mathrm{eV}$ ) are represented by a thermal maxwellian. This spectral shape is quite typical of all water-cooled, mixed-spectrum research and commercial power reactors. The explanation for this spectrum is that the very highest neutron energies produced by fission in the uranium fuel are relatively unaffected. At lower neutron energies, the strong moderation due to neutron scattering from hydrogen in the coolant water leads to a $1 /$ E slowing down spectrum (thus looking flat when multiplied times energy). This process continues until the thermal motion of atoms in the moderator is equal to the neutron energy at which point a neutron can gain energy in a collision as well as lose it. This results in a thermal maxwellian distribution centered at the temperature of the coolant. At room temperature, the average neutron energy is $0.0253 \mathrm{eV}(3 / 2 \mathrm{kT})$; this energy is also generally used to define thermal neutron cross sections and corresponds to the well-known thermal neutron velocity of $2200 \mathrm{~m} / \mathrm{s}$. In a fission reactor, the coolant temperature may be 
considerably above room temperature, and hence the peak will have a higher mean energy.

\section{Particle Accelerator Neutron Sources}

Neutrons can also be produced from nuclear reactions induced by energetic charged particles. Many different reactions can be used. One of the most common sources is the $14 \mathrm{MeV}$ neutron source which is illustrated in Figure 5 by the Rotating Target Neutron Source (RTNS)II, which operated at Lawrence Livermore National Laboratory (CA) until it was closed in 1987.[5] This type of source produces neutrons from the interaction of deuterium and tritium. This reaction releases a lot of energy and is thus the favorite choice for fusion reactors. In fact, only a few tens of kilovolts are needed to produce $14 \mathrm{MeV}$ neutrons, and this can be accomplished by small, compact $d-t$ neutron generator tubes. The primary interest in this type of source is to simulate fusion reactor neutron spectra, although compact neutron generator tubes are also widely used for neutron activation, for example, to log oil wells.

Another type of source that has been proposed to simulate fusion spectra is based on the $\mathrm{Be}$ or $\mathrm{Li}(d, n)$ reaction. In this case, a deuterium beam of particles is stopped in a thick $\mathrm{Be}$ or $\mathrm{Li}$ target. If the deuterium energy is 35 to $40 \mathrm{MeV}$, then the average neutron energy will be about $14 \mathrm{MeV}$, as illustrated by the Li(d,n) spectrum shown in Figure 6, which further compares the spectrum expected at a future fusion power reactor (DEMO) with that at various accelerator-based neutron sources.[6]

Some interest has also been raised in spallation neutron sources that produce neutrons from spallation reactions where proton beams at hundreds of $\mathrm{MeV}$ are stopped in large targets. This is also illustrated in Figure 6 and Figure 7 for the Los Alamos Radiation Effects Facility (LASREF) [7] at Los Alamos National Laboratory (NM) and the Intense Pulsed Neutron Source (IPNS) [8] at Argonne National Laboratory (IL). Note that most of the neutrons at spallation sources peak around $1 \mathrm{MeV}$; however, LASREF has a 
weak tail extending to $800 \mathrm{MeV}$ and IPNS to $450 \mathrm{MeV}$ since these are the energies of the incident proton beams. Figure 6 also illustrates a proposed $H(t, n)$ source consisting of a tritium beam on a water target.[6]

One of the limitations of particle neutron sources at present is that the neutron fluxes are generally much lower than in fission reactors. Another significant problem is that the space available for irradiating materials is generally much smaller in an acceleratorbased neutron source. A listing of the neutron fluxes and volumes at some of the materials research facilities is given in Table I. (This is also illustrated for radiation damage parameters later in Figure 21.)

\section{Neutron Flux and Spectral Measurements}

To calculate dpa during a neutron irradiation, it is necessary to determine the neutron flux and energy spectrum. As illustrated in Figure 1, exposure units such as dpa allow us to directly compare materials effects measured at very different irradiation facilities, such as mixed-spectrum reactors, fast reactors, and particle accelerators. If we restrict ourselves to one fixed irradiation condition, then knowledge of the neutron flux and energy spectrum is of secondary interest. However, measurement of the neutron exposure is required to allow us to determine an appropriate measure of the irradiation exposure that can be compared with other irradiation conditions.

Many computer codes have been developed to calculate the neutron flux spectrum from first principles. These are referred to as neutronics codes or transport codes, which follow the course of neutrons through various materials. At the point of generation, the neutron flux spectra can be determined quite precisely from the fission process. However, operating fission reactors become quite complicated when one takes into account moderators, reflectors, control rods, coolant, and other structural materials, as well as the geometry of the facility. As the facility becomes more complicated or as we transport neutrons through more materials, the reliability of the 
calculations decreases. It is thus highly desirable to use a technique to measure the flux spectrum to check on these calculations.

Several methods are used to measure the neutron flux spectrum, depending on the type of facility. The most widely used technique for fission reactors is referred to as the multiple activation technique. Neutron activation refers to the process whereby a neutron reacts with a target nucleus and transmutes it to a different isotope. These reactions are given symbolically in parentheses where the first entry is the incoming particle and the second entry is the outgoing particle. The notation $A(n, p) B$ thus means that nucleus $A$ combined with a neutron, emitted a proton, and was transmuted to nucleus $B$ in the process. The $(n, p)$ reaction will thus lower the atomic number by one, for example, ${ }^{54} \mathrm{Fe}(n, p){ }^{54} \mathrm{Mn}$. Other reactions of most interest include $(n, g a m m a)$, (n, fission), ( $n$,alpha), and $(n, 2 n)$. In many cases, the product nucleus is radioactive and can be measured to determine the number of such reactions that occurred. Each type of reaction has a different dependence on neutron energy, as illustrated in Figure 8. Occurring at thermal neutron energies ( $n$,gamma) and (n,fission) reactions thus have no energy threshold. Fission reactions can only occur for the actinides such as $U, N p$, and Pu. Capture gamma reactions are the most common source of nuclear transmutation and are widely used to produce radioactive isotopes for commercial purposes.

To measure the neutron flux spectrum, it is possible to simultaneously irradiate a number of different materials and look at the activation products that are produced. Each of the products yields information about a different region of the neutron energy spectrum. For example, (n,gamma) reactions are sensitive mainly to the thermal neutron flux; ( $n, p)$ reactions measure above $1 \mathrm{MeV}$; ( $n$,alpha) above 4-5 MeV; and $(n, 2 n)$ above about $10 \mathrm{MeV}$. The epithermal energy region (1/E spectrum) can be determined either by covering thermal flux monitors with a strong thermal neutron absorber, such as cadmium, or by using resonance detectors, which have a strong reaction rate at a very narrow energy region, as illustrated by the sharp peaks for the 
(n,gamma) reaction in Figure 8.

The rate at which a nuclear reaction occurs is defined as the cross section given in units of barns where 1 barn $=10^{-24} \mathrm{~cm}^{2}$. This can be thought of as the area of interaction between an incoming neutron and a target nucleus. Activation products are most easily measured by gamma energy analysis since each radioactive isotope emits a unique combination of gamma rays. The measured activity per atom is then corrected for radioactive decay, both during and after irradiation, to determine the saturated reaction rate. Other corrections that may be needed include burnin and burnout of various isotopes, neutron and gamma self-absorption, and fission yields. Mathematically, the saturated reaction rate is equal to the integral over neutron energy of the energy dependent neutron activation cross section times the energy dependent neutron flux. If we measure a number of reaction rates in the same neutron spectrum, then we have a series of integral equations all of which have a common neutron flux spectrum as the solution. This coverage of the neutron spectrum is illustrated for a mixed-spectrum fission reactor in Figure 9.

\section{Neutron Spectral Adjustment}

A number of computer codes have been developed over the years to solve the integral reaction rate equations. The earliest codes, such as SANDII [9], used iterative techniques to vary the flux looking for a minimum deviation between measurement and calculations. More recent codes, such as FERRET [10] and STAY'SL [11], use a generalized least-squares technique. These codes allow us to take into account all known uncertainties and their correlations in the measured reaction rates, nuclear cross sections, and starting flux spectrum. The output flux spectrum thus also provides a full covariance matrix to track all uncertainties.

Neutron activation cross sections and their uncertainties are generally available from the Evaluated Nuclear Data File (ENDF) [12]. These ENDF data are available from the 
National Neutron Cross Section Center at Brookhaven National Laboratory either electronically or by magnetic tape. Many of the cross sections have undergone integral testing in various standard neutron fields. Testing has also been performed by comparison with other active neutron measurement techniques such as time-offlight (TOF) spectroscopy, [13-15] has been used to directly measure the neutron flux spectrum at particle accelerator-based neutron sources. However, it may not be possible to measure the spectrum very close to the target where most materials irradiations are performed to maximize the fluence. Hence, activation techniques have also been used to characterize accelerator-based neutron irradiation facilities.

The input neutron spectra for spectral adjustment are ideally taken from neutronics calculations, which can take into account the known source spectra, moderators, reflectors, sample materials, etc., that may be present in a given reactor position. This is where the name "adjustment" comes from since we are intending to adjust a calculated neutron spectrum to determine the best fit to the data. Having a good starting spectrum is important for several reasons. First of all, the least-squares technique by definition has poor energy resolution. That is, most reactions have rather broad energy responses and hence the technique is not sensitive to fine detail in the neutron spectrum; such detail can only come from physics calculations. Secondly, the technique is not very sensitive to intermediate energy neutrons. Many reactions will define the thermal, epithermal, and fast neutron energy regimes; however, few reactions are sensitive to the energy region from about 0.01 to 1000 $\mathrm{keV}$.

Unfortunately, many instances occur where good neutronics calculations are not available, often due to time and funding limitations. Even in these cases, it is possible to determine a crude neutron spectrum using only the integral activation data. One possibility is to use spectra from other facilities that are believed to be similar. In any case, one can determine the reliability of the technique in several different ways. One that has already been mentioned is to compare the results to a known standard such 
as a 252-Cf source or an accelerator neutron spectrum determined by time-of-flight spectroscopy. The other possibility is to perform sensitivity studies where various input spectra are tried to see what the effect will be on the output spectrum. Input flux uncertainties can also be varied to study their impact on the output. In these ways, it is always possible to get a reliable estimate of the neutron flux spectra from integral data. However, one must bear in mind the basic limitations of the techniques that were mentioned earlier, specifically regarding intrinsically poor energy resolution and the lack of sensitivity in some energy regions.

The choice of neutron activation reactions is governed by many factors, including the length of irradiation vs. the decay time of the product isotope, and the amount of activity generated vs. the available flux and space provided for the activation materials. Generally, the best spectral measurements require rather short irradiations (1 to 3 days) with rapid gamma counting of the products. On the other hand, it is possible to monitor the progress of very lengthy irradiations over many years by normalizing a few key reactions to a previously determined neutron spectrum.

Although it is not always possible to obtain highly accurate information about the details of a neutron spectrum, the multiple activation technique does provide rather accurate integral values by keeping track of all uncertainties and their covariances. Hence, it is routinely possible to obtain accuracies of 10 to $20 \%$ for integral quantities such as the thermal flux, flux $>0.1 \mathrm{MeV}$, flux $>1 \mathrm{MeV}$, etc., even though we do not know every wiggle in the spectrum This level of accuracy can also be carried through for subsequent transmútation and damage calculations since these are also integral quantities.

An example of a neutron spectral adjustment is shown in Figure 10 and Table $\|$ for the Fast Flux Test Facility. Note that with rather small spectral adjustments, the fit to the data is improved substantially and that we are able to get excellent internal consistency for many different activation reactions. 


\section{Nuclear Transmutation Effects}

One of the objectives of knowing the neutron flux spectra for a given irradiation is to permit reasonable estimation of other nuclear transmutation effects such as activation, gas production, and changes in atomic concentrations. It is well known that nuclear transmutation will lead to measurable changes in the propertities of materials. Accurate knowledge of such effects is important for predictions of materials performance in high fluence irradiations, such as fusion power reactors. Once we have determined the neutron flux spectrum, then it is straightforward to calculate gas production and other transmutation rates by integrating the appropriate neutron cross section from ENDF over the adjusted neutron flux spectrum. For example, the STAY'SL computer code has been modified to routinely provide recommended reaction rates as part of the output. Such information is used to determine nuclear burnin and burnout effects as well as transmutation to other atomic species.

For very lengthy irradiations, transmutation calculations may become very complicated since a long chain of transmutation may exist from element $A$ to $B$ to $C$, etc. Another complication is that neutron reaction cross sections are not well known for most radioactive isotopes, simply because it is very difficult to handle and to measure reaction rates for radioactive materials. Hence, computer codes, such as REAC [16], have been developed to calculate such effects using neutron cross sections, which are calculated from basic nuclear reaction theory and/or semi-empirical models. Such calculations may not be wholly reliable, but they provide reasonable estimates for activation of nuclear components and waste material. Many such calculations of transmutation have been published for fusion power reactor materials. [17-18]

It has also been pointed out [19] that the primary proton and alpha recoil atoms produced by neutron bombardment may also cause activation of materials. This has led some to question the concept of low activation materials for fusion reactors since materials, such as vanadium, which are not activated very much by neutrons, may in 
fact be activated by the proton and alpha recoils. Measuring such effects is extremely difficult since it may not be possible to distinguish between such activations and neutron reactions with impurities in the starting materials.

\section{Special Thermal Neutron Helium Production}

The high flux of $14 \mathrm{MeV}$ neutrons in fusion devices produces substantially more hydrogen and helium than is produced during comparable irradiations in fission reactors. For this reason various techniques have been developed to enhance helium production during fission reacior irradiations to more accurately simulate future fusion reactor conditions. Hydrogen is not thought to be a problem due to the high mobility of hydrogen in most materials. However, helium is trapped in voids and leads to substantial swelling. [20]

One such helium production reaction of special note is the ${ }^{58} \mathrm{Ni}(\mathrm{n}, \gamma)^{59} \mathrm{Ni}(\mathrm{n}, a)^{56} \mathrm{Fe}$ reaction.[21,22] In a mixed-spectrum reactor, the high thermal neutron flux will quickly transform ${ }^{58} \mathrm{Ni}$ to ${ }^{59} \mathrm{Ni}$ which in turn readily produces helium (alphas). This effect is illustrated in Figure 11. Although the effect is not linear with exposure, due to the time required to breed ${ }^{59} \mathrm{Ni}$, spectral tailoring has been employed to help smooth out the process. [23] Recent experiments [24] have also used ${ }^{59} \mathrm{Ni}$ doping of starting materials, which is equally effective at producing helium in fast reactors. The nickelhelium trick is especially effective for nickel-bearing alloys such as stainless steel since we are not required to add unnatural atomic additions to the matrix. It should also be noted that the ${ }^{56} \mathrm{Fe}$ recoil from the ${ }^{59} \mathrm{Ni}(\mathrm{n}, \alpha)^{56} \mathrm{Fe}$ reaction has an energy of $340 \mathrm{keV}$, a very high energy indeed (it would take a $20 \mathrm{MeV}$ neutron to produce a recoil of this energy). This energetic recoil also produces significant displacement damage in the ratio of 1 dpa for every 567 appm helium [22] (See Table III).

Other reactions that are known to enhance helium production include doping with ${ }^{10} \mathrm{~B}$ or ${ }^{6} \mathrm{Li}$ or the tritium trick, since tritium decays to ${ }^{3} \mathrm{He} .[25]$ This tritium effect has 
recently been used in fast reactors to enhance helium production in vanadium alloys.[26] It has also been shown that a thermal neutron-induced enhancement of helium in copper occurs from the ${ }^{63} \mathrm{Cu}(n, y)^{64} \mathrm{Cu}(\beta)^{84} \mathrm{Zn}(n, y)^{65} \mathrm{Zn}(n, a)^{62} \mathrm{Ni}$ reaction. [27] The copper effect is not as dramatic as for nickel since an additional step is required to produce the ${ }^{65} \mathrm{Zn}$ and, at present, there is little interest in zinc-bearing alloys where the effect would be comparable to nickel-bearing alloys. The copper effect is illustrated for HFIR in Figure 12. It has also been suggested that a similar weak effect may be present for iron from the ${ }^{54} \mathrm{Fe}(n, y)^{55} \mathrm{Fe}(n$, alpha) reactions; however, further testing is needed to substantiate this process.[28]

Influence of Transmutation on Materials Property Effects

The transmutation of elements in a mixed-spectrum reactor can be quite dramatic for those elements that have substantial thermal neutron cross sections. For example, gold will transmute to mercury at the rate of about $13 \%$ per month in HFIR (too bad we cannot go the other way!). Recently, we have done transmutation calculations for some suggested engineering materials containing Mo, W, Re, and $\mathrm{Nb}$.[29] Figure 13 illustrates the transmutation of such materials in HFIR and a fusion power reactor, STARFIRE. The high transmutation levels demonstrate that it is thus quite important to consider such effects on material properties since changes in the atomic composition below the $1 \%$ levels are known to have dramatic effects on materials' propertities.

\section{Atomic Recoil Spectra}

One of the most important effects of neutron irradiation on materials is that atoms are displaced from their lattice sites due to their recoil energy following a nuclear reaction. Such atomic displacements are referred to as displacements per atom or dpa. The initial interaction of a neutron with an atom can lead to a significant recoil energy; such events are referred to as primary knock-on atoms (PKA). The PKA energy 
distribution is determined by the sum of recoils from all of the possible neutron reactions. Each type of reaction produces a different recoil energy distribution depending on the masses involved, the incident neutron energy, and the angle between the incident neutron direction and the final recoil direction. For example, the $(n, y)$ capture reaction leads to rather small recoil energies since the photon is massless and cannot carry away very much momentum. However, charged particle reactions such as $(n, p)$ or $(n, a)$ can lead to very high recoil energies determined mainly by the masses of the target and product atoms. Obviously, light elements will recoil with more energy than heavy elements due to the need to conserve momentum and energy in all such reactions. Examples of recoil spectra for various reactions are shown for nickel in HFIR in Figure 14. In fission reactors, most of the recoils are caused by elastic or inelastic scattering and thus have rather low recoil energies, as can be seen in the figure. Most of the nickel recoils in HFIR are thus below $100 \mathrm{keV}$, although a small fraction of recoils extend up to about $2 \mathrm{MeV}$. One popular way to illustrate differences in recoil spectra between different facilities is to show the integral above the energy plotted, as shown in Figure 15. The $50 \%$ point thus means that half the recoils are above that energy.

The calculation of primary recoil spectra is facilitated by computer codes such as SPECTER.[30] In the previous talk by Mark Robinson, there was some discussion of the recoils for a given neutron energy (e.g., $1 \mathrm{MeV}$ neutron incident on nickel). However, when we irradiate nickel in a fission reactor, we must integrate all possible reactions 'over all neutron energies. The SPECTER code does this by using a built-in library of recoil energy spectra over a 100-point grid as a function of neutron energy. These libraries thus typically store 10,000 data points to fully describe all possible PKA spectra. Thus, the code only needs the neutron energy spectrum as input and can then calculate spectral-averaged recoil spectra, as shown in Figures 14 and 15.

Displacement per atom calculations start with the primary recoil spectra determined from the neutron interactions with the matrix atoms. It is important to note that each 
primary recoil atom is capable of displacing a large number of secondary atoms. The number of secondary displacements is determined by two factors: the total amount of energy available and the energy required to displace an atom. The recoiling primary atom will mainly slow down due to electronic interactions. The total energy dissipated in this slowing down process is referred to as Kerma. A small part of this energy will be available for displacing other atoms through nuclear collisions. This distribution of electronic vs. nuclear stopping power is usually calculated by the Lindhard theory [31]. Thus for any given combination of recoil atom and matrix material, the nuclear stopping energy can be determined. This energy is referred to as the damage energy or $T_{\text {dam }}$. The expression $0.8 \mathrm{~T}_{\text {dam }} / 2 \mathrm{Ed}$, where $\mathrm{Ed}$ is the energy required to displace an atom from its lattice site, finally gives the total number of secondary displacements. The total displacement cross section in barns thus represents the total number of both primary and secondary displaced atoms generated by an incident neutron. Multiplying the dpa cross section times the total neutron fluence will then give the total number of displaced atoms for a given irradiation. Although we are talking about displaced atoms, note that dpa is a calculated parameter and does not represent the actual number of crystal lattice: defects that have been created. Defect production will be discussed at length in other chapters.

The fundamental theory behind the SPECTER computer code is described in Reference 32. Displacement cross sections are calculated for each type of nuclear reaction, as shown in Figure 16. At low energies, it is now clear that only $(n, y)$ and elastic scattering contribute to the displacement damage. The only exception to this is for reactions such as $(n, a)$ that occur at thermal neuton energies, such as for ${ }^{6} \mathrm{Li},{ }^{10} \mathrm{~B}$, or ${ }^{59} \mathrm{Ni}$ (see Table III). Recoils from the $(n, r)$ reaction are caused by photon emission. The net recoil energy is determined by summing over all possible photons (some may go up to 8 to $10 \mathrm{MeV})$ using the relationship $E_{r}(e V)=E_{0}{ }^{2} /(A+1) m_{0} c^{2}=536.7 E_{o}{ }^{2}$ $\|(A+1)$ where $A$ is the mass of the target nucleus and $E_{0}$ is the gamma energy in $\mathrm{MeV}$. Since many isotopes also undergo beta decay following neutron capture gamma emission, this effect has also been included in Table IV which lists the true average 
and effective recoil energies following ( $n$,gamma) reactions for a number of elements. Clearly, these recoil energies are quite small and generally are not the major contributors to dpa cross sections except in highly thermal neutron spectra.

\section{SPECTER Computer Code}

The SPECTER computer code provides a convenient way to obtain damage parameters for a variety of elements and compounds in a specified neutron energy spectrum. The user need only specify the neutron spectrum. The code then integrates over the libraries described previously to give the spectral-averaged results of interest. If an irradiation time is given, then results will be quoted at the neutron fluence specified. The output includes spectral-averaged Kerma (total energy), gas production, displacement damage, and PKA recoil distributions. An example of the dpa calculations are listed in Table V. Dpa calculations are listed separately for each type of reaction and then summed to give the net displacement damage. Other computer codes are also widely used such as NJOY [33]. However, some of the advantages of SPECTER are that since the basic neutron reaction calculations have already been performed to create the SPECTER libraries, the user does not need access to ENDF data files, and the code is compact and can be run in minutes on a PC-level computer.

\section{Compound Materials}

Displacement damage in compound materials is fundamentally the same as for pure elements in that the PKA energy distributions are the same since they arise purely from the interactions of neutrons and individual atoms. However, the secondary displacements are quite different since we must now consider all possible combinations of primary recoil and matrix atoms. For example, in the case of $\mathrm{LiO}_{2}$, recoiling $\mathrm{Li}$ atoms will interact with both $\mathrm{Li}$ and $\mathrm{O}$ atoms; the frequency only depends on the atomic ratios. Computer codes such as SPECOMP $[34,35]$ have been developed to consider these interactions. The SPECOMP code starts with the PKA 
libraries contained in SPECTER as the primary damage state. Lindhard damage energy calculations are then performed for all possible combinations of recoil and matrix atom, as shown in Figure 17. All of these combinations can then be added up according to their atomic abundance to determine the total dpa cross section. However, it is important to note that $\mathrm{dpa}$ in this case refers to the displacement of any type of atom in the compound rather than one specific atomic species. A further complication is that the displacement energy threshold may be different for each element in a compound.

One of the main problems with defining dpa cross sections for compound materials is that displacement threshold energies are not well known. In a pure element, this problem is not so significant since we can always simply renormalize the cross section using the relationship of $0.8 \mathrm{~T}_{\mathrm{dem}} / 2 \mathrm{Ed}$ (i.e. - dpa is proportional to $1 / \mathrm{E}_{\mathrm{d}}$ ). However, for a compound, such renormalization is not possible, and a change in any of the threshold energies requires a completely new calculation of the dpa cross section.[35]

It is important to note that the results of a SPECOMP calculation may be quite different than might be expected from simply adding up elemental damage. This is illustrated for $\mathrm{Li}_{2} \mathrm{O}$ in Figure 18. Examples of calculations for various compounds are listed in Table VI.

\section{Conclusions and Future Directions}

It is important to note that the concept of dpa is only a crude indicator of the complex interactions that will be produced by neutron irradiations. Most of the displaced atoms will freely migrate and recombine with vacancies. Recombination strongly depends on temperature due to the mobility of the interstitial atoms; hence, experiments that attempt to measure total displaced atoms (e.g., by resistivity changes) are generally conducted at liquid helium temperatures of $4^{\circ} \mathrm{K}$. The calculation of dpa is only indicative of the total initial energy that is available to 
produce damage to ihe matrix - not the final, permanent damage. Many models have been developed to follow subsequent interactions to the formation of more permanent damage such as defect clusters, cascade loops, etc., and such effects will be discussed in subsequent chapters. The reason that the dpa parameter has been so successful as a correlation parameter is that dpa is proportional to the total energy available for producing defects which in turn is generally proportional to the final material defects that remain in the crystal lattice.

Some success has recently been achieved with models such as freely migrating interstitials [36] to replace dpa. Computer models consistently show that lower energy recoils are more effective at producing stable defects than high energy recoils. An example of two such models is shown in Figure 19. The $\mathrm{H}_{\bmod }$ curve represents computer calculations of freely migrating interstitials following neutron bombardment.[36] The Wmod curve is taken from the survival of defects following heavy ion irradiaitons.[37] Both models can be used to derive modified displacement damage cross sections, as shown in Figure 20. Note that all such models produce smaller damage cross sections since dpa is a measure of the maximum damage possible. However, the models obviously change the ratio of defects at lower and higher neutron energies, thereby producing some interesting spectral effects such as an enhancement of thermal neutron damage relative to fast damage. However, such models are still in the developmental stage and are not yet universally accepted. Some experiments are in progress to attempt to study such effects, for example, by looking for enhanced damage from thermal neutrons.

Neutron sources, such as fission reactors and particle accelerators, can be viewed in terms of their ability to produce radiation damage in materials, as shown in Figure 21, which compares dpa rate vs. helium production rate for various facilities. The figure is drawn from the point of view of fusion materials research where the helium to dpa ratio in iron or stainless steel is about 10/1. It is apparent that few facilities match the fusion conditions, and this is why researchers have looked at tricks such as the 
thermal helium production in nickel for mixed spectrum reactors. The fusion community has also long advocated [6] using particle accelerator devices such as Li $(d, n)$ which can generate fusion-like damage both in terms of neutron flux as well as helium to dpa ratios. Using higher energy accelerators is not without problems in that neutron cross sections for activation, transmutation, and radiation damage are generally not well known above about $14 \mathrm{MeV}$ due to measurement problems. This problem becomes more significant with higher energy accelerators such as spallation devices; however, one can argue that the fraction of neutrons at very high energies is not high enough to cause severe differences with lower energy irradiations. In any case, more effort is needed to develop neutron cross sections for all applications at higher neutron energies.

In conclusion, this chapter has attempted to give an overview of neutron irradiation facilities, flux and spectral measurements, atomic recoil spectra, radiation damage calculations, and the interconnections between all of these subjects. Many years have been spent developing these techniques, and, in general, we now have extensive capabilities to characterize irradiation facilities both in terms of neutron flux spectra and radiation damage. Such data are widely used to correlate materials effects between different types of irradiation facilities and to predict damage to fusion reactor materials. However, more development is needed in each of the following areas. Neutron interaction cross sections are needed to improve activation and spectral adjustment techniques, to improve the prediction of materials activation for reactor components and waste materials, and to improve the estimation of radiation damage phenomena. If higher energy particle accelerator neutron sources are used, then neutron cross sections and damage cross sections are especially needed at energies above $20 \mathrm{MeV}$. In the radiation damage area, new models are being developed to replace the dpa concept with energy, material, and property-dependent damage parameters. However, at present, dpa remains the best exposure parameter for comparisons between different types of irradiation facilities. For fusion materials studies, work is needed to improve the extrapolation of materials effects from non- 
fusion spectra at very different flux levels to conditions expected at an operating fusion power reactor. 
Table I: Neutron Fluxes at Selected U.S. Materials Irradiation Facilities Maximum values are listed near core center at full power.

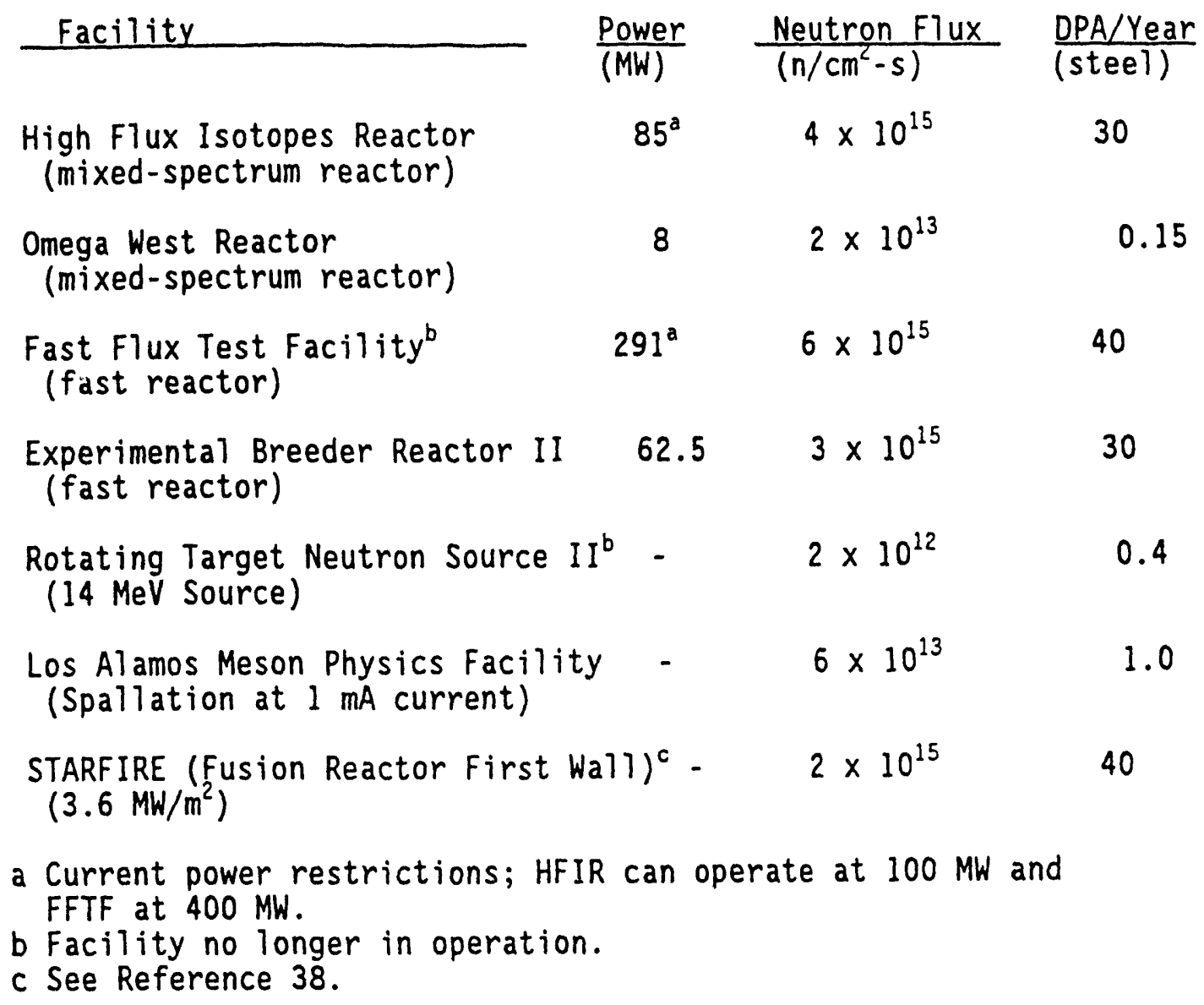


Table II: STAY'SL Spectral Adjustments for FFTF/MOTA-1G

$$
\text { Position: }-1.1 \mathrm{~cm} \quad \underline{\mathrm{CHI}}{ }^{2}=0.45
$$

Comparison of Reaction Rates (atom/atom-s)

\begin{tabular}{|c|c|c|c|c|c|c|c|c|}
\hline \multirow{3}{*}{$\begin{array}{l}\text { Measured } \\
\\
2.32 E-10 \\
3.92 E-11 \\
8.33 E-10 \\
3.81 E-11 \\
1.88 E-13 \\
4.36 E-11 \\
7.44 E-13 \\
3.39 E-12 \\
1.95 E-09 \\
7.92 E-09 \\
8.12 E-09\end{array}$} & \multirow[b]{2}{*}{$\begin{array}{r} \pm \% \\
5 \\
5 \\
5 \\
5 \\
5 \\
8 \\
10 \\
5 \\
7 \\
6 \\
6\end{array}$} & Calculated & Diff. & \multirow[b]{2}{*}{$\begin{array}{l}\text { STAY'SL } \\
\\
2.32 E-10 \\
3.96 E-11 \\
8.35 E-10 \\
3.62 E-11 \\
1.75 E-13 \\
4.96 E-11 \\
8.68 E-13 \\
3.75 E-12 \\
1.92 E-09 \\
8.11 E-09 \\
7.98 E-09\end{array}$} & \multirow[b]{2}{*}{$\begin{array}{l}\text { Diff. } \\
\% \\
0.0 \\
1.2 \\
0.3 \\
-5.1 \\
-7.2 \\
13.8 \\
16.6 \\
10.7 \\
-1.8 \\
2.3 \\
-1.7\end{array}$} & \multirow[b]{2}{*}{$\begin{array}{l}\text { Reaction } \\
\text { C059(N,G)C060 } \\
\text { FE58(N,G)FE59 } \\
\text { NB93(N,G)NB94 } \\
\text { FE54(N,P)MN54 } \\
\text { CU63(N,A)CO60 } \\
\text { NI58(N,P)CO58 } \\
\text { NI60(N,P) CO60 } \\
\text { TI46(N,P) SC46 } \\
\text { NP237(N, FISSION) } \\
\text { PU239(N, FISSION) } \\
\text { U235(N,FISSION) }\end{array}$} & \multicolumn{2}{|c|}{ Energy Range, MeV } \\
\hline & & $\begin{array}{l}5.03 E-10 \\
5.36 E-11 \\
9.94 E-10 \\
3.68 E-11 \\
2.07 E-13 \\
4.97 E-11 \\
1.04 E-12 \\
4.39 E-12 \\
1.72 E-09 \\
8.77 E-09 \\
8.92 E-09\end{array}$ & $\begin{array}{r}117 \\
37 \\
19 \\
-3 \\
10 \\
14 \\
40 \\
30 \\
-12 \\
11 \\
10\end{array}$ & & & & $\begin{array}{l}1.00 E-04 \\
2.80 E-04 \\
5.75 E-04 \\
2.00 E+00 \\
4.50 E+00 \\
1.60 E+00 \\
4.50 E+00 \\
3.30 E+00 \\
3.60 E-01 \\
7.60 E-04 \\
5.75 E-04\end{array}$ & $\begin{array}{l}3.60 E-01 \\
5.50 E-01 \\
5.00 E-01 \\
6.70 E+00 \\
1.10 E+01 \\
6.70 E+00 \\
1.00 E+01 \\
9.00 E+00 \\
3.30 E+00 \\
2.00 E+00 \\
1.60 E+00\end{array}$ \\
\hline & & & 43 & & 8.2 & & & \\
\hline
\end{tabular}


Table III: Calculation of Damage for ${ }^{59} \mathrm{Ni}$ Helium Reactions

$$
\begin{aligned}
& { }^{58} \mathrm{Ni}(n, \gamma){ }^{59} \mathrm{Ni}(n, \alpha){ }^{56} \mathrm{Fe} \\
& Q-V a l u e=5097 \mathrm{keV} \\
& E(56-\mathrm{Fe})=340 \mathrm{keV}=(4 / 60) \times Q \\
& E(\alpha)=4757 \mathrm{keV}=(56 / 60) \times Q
\end{aligned}
$$

Damage Energy (Lindhard Thenry)

$$
\begin{aligned}
& T_{\text {dam }}(56-\mathrm{Fe})=170.0 \mathrm{keV} \\
& T_{\text {dam }}(\alpha)=6.2 \mathrm{keV} \\
& \text { Total } T_{\text {dam }}=176.2 \mathrm{keV} \\
& \text { DPA }=0.8 T_{\text {dam }} / 2 \mathrm{E}_{\text {d } 1}=1762 \mathrm{dpa} / \mathrm{he} 1 \mathrm{ium} \\
& \mathrm{He}(\mathrm{appm}) / \mathrm{dpa}=10^{5} / 1762=567 \mathrm{He}(\text { appm }) / \mathrm{dpa}
\end{aligned}
$$




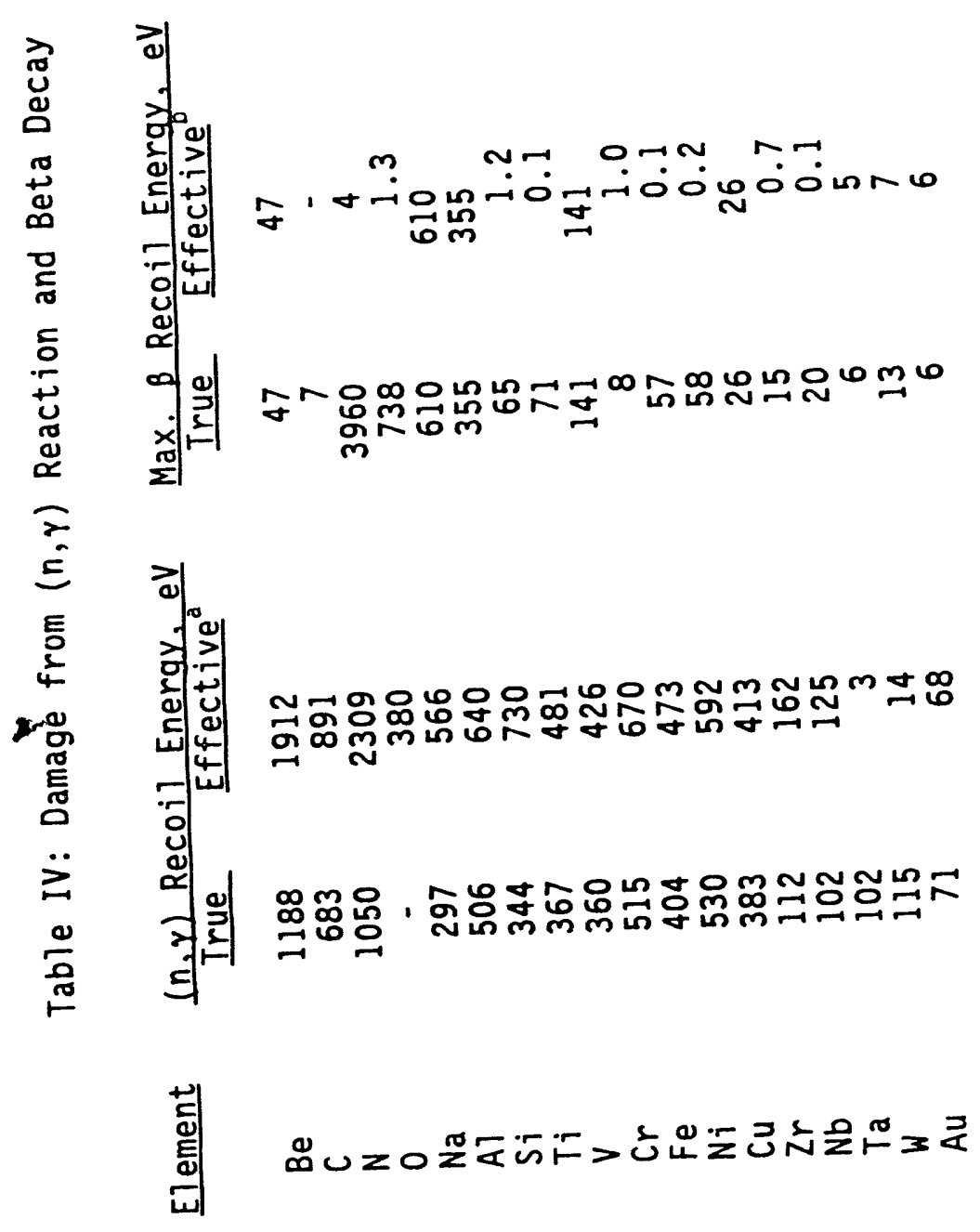


Table V: Example of SPECTER Output for FFTF/MOTA - Midplane MOTA-2A

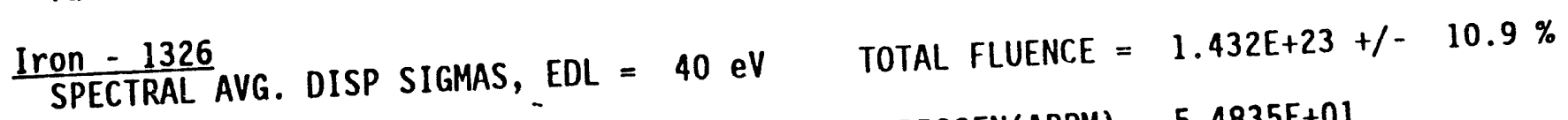
$D P A=4.1343 E+01 \quad H E L I U M(A P P M)=2.8621 E+00 \quad \operatorname{HYDROGEN}(A P P M)=5.4835 E+01$

ELASTIC INELST $(\mathrm{N}, 2 \mathrm{~N}) \quad(\mathrm{N}, 2 \mathrm{~N}) \mathrm{P} \quad(\mathrm{N}, 2 \mathrm{~N}) \mathrm{SUM} \quad \mathrm{CH} \quad \mathrm{CH} 2 \quad$ SUM

$\begin{array}{llllllllll}2.5530 E+02 & 3.2810 E+01 & 8.8613 E-03 & 2.7826 E-02 & 3.6685 E-02 & 2.0248 E-01 & 2.5302 E-02 & 2.8838 E+02 & \text { BARNS }\end{array}$

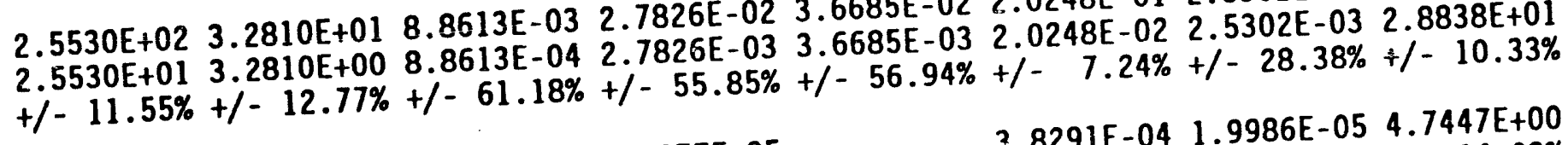

4.6493E+00 9.4899E-02 6.3657E-06 1.9277E-05 $+/-14.31 \%+/-17.12 \%+/-60.67 \%+/-55.62 \%$

$3.8291 \mathrm{E}-04 \quad 1.9986 \mathrm{E}-05 \quad 4.7447 \mathrm{E}+00$

NUCLEAR - BARNS

CAPTURE GAMMA DAMAGE $=3.1571 \mathrm{E}-02 \mathrm{KEV}-\mathrm{B}+/-11.62 \% \quad \mathrm{CS}=1.1091 \mathrm{E}-02$ BARNS, TGAM $=395.0 \mathrm{EV}$

TOTAL DPA CROSS-SECTION $=2.8869 E+01 \mathrm{KEV}-\mathrm{B} ;$ OR $2.8869 \mathrm{E}+02$ BARNS

Nickel - 1328

SPECTRAL AVG. DISP SIGMAS, EDL $=40 \mathrm{eV}$

TOTAL FLUENCE $=1.432 E+23+/-10.93 \%$

$D P A=4.8461 E+01 \quad H E L I U M(A P P M)=4.2216 E+01 \quad H Y D R O G E N(A P P M)=7.9895 E+02$

ELASTIC INELST (N,2N) (N,2N)P $\quad(N, 2 N) S U M \quad C H 1 \quad$ CH2 $\quad$ SUM

$\begin{array}{lllllllll}3.1671 E+02 & 1.8644 E+01 & 1.2589 E-02 & 2.5892 E-01 & 2.7151 E-01 & 1.8594 E+00 & 4.4731 E-01 & 3.3793 E+02 & \text { BARNS } \\ 3.1671 E+01 & 1.8644 E+00 & 1.2589 E-03 & 2.5892 E-02 & 2.7151 E-02 & 1.8594 E-01 & 4.4731 E-02 & 3.3793 E+01 & \text { KEV-BARNS }\end{array}$ $+/-11.43 \%+/-9.08 \%+/-56.04 \%+/-26.52 \%+/-27.34 \%+/-10.02 \%+/-9.52 \%+/-10.77 \%$

$7.9783 \mathrm{E}+00 \quad 5.1133 \mathrm{E}-02 \quad 8.9378 \mathrm{E}-062.2227 \mathrm{E}-04$ $+/-19.61 \%+/-12.11 \%+/-55.00 \%+/-25.30 \%$

$5.5790 E-032.9479 E-048.0355 E+00$
$+/-6.82 \%+/-6.39 \%+/-19.47 \%$

CAPTURE GAMMA DAMAGE $=4.6662 \mathrm{E}-02 \mathrm{KEV}-\mathrm{B}+/-22.59 \% \quad \mathrm{CS}=1.7558 \mathrm{E}-02$ BARNS, $\mathrm{TGAM}=491.0 \mathrm{EV}$

TOTAL DPA CROSS-SECTION $=3.3840 E+01 \mathrm{KEV}-\mathrm{B} ;$ OR $3.3840 E+02$ BARNS 
Table VI: SPECOMP Results for Various Compounds and Facilities Comparison of spectral-averaged dpa cross sections (barns)

\begin{tabular}{|c|c|c|c|c|c|}
\hline Compound & & $14 \mathrm{MeV}$ & Starfire & HFIR & EBRII \\
\hline $\mathrm{Ii}_{2} \mathrm{O}$ & $\begin{array}{l}\text { SPECOMP } \\
\text { Sum }\end{array}$ & $\begin{array}{r}1040 \\
728\end{array}$ & $\begin{array}{l}754 \\
517\end{array}$ & $\begin{array}{l}2410 \\
2321\end{array}$ & $\begin{array}{l}939 \\
636\end{array}$ \\
\hline $\mathrm{LiAlO}_{2}$ & $\begin{array}{l}\text { SPECOMP } \\
\text { Sum }\end{array}$ & $\begin{array}{l}1648 \\
1336\end{array}$ & $\begin{array}{l}978 \\
777\end{array}$ & $\begin{array}{l}1120 \\
1050\end{array}$ & $\begin{array}{r}1031 \\
808\end{array}$ \\
\hline $\mathrm{Al}_{2} \mathrm{O}_{3}$ & $\begin{array}{l}\text { SPECOMP } \\
\text { Sum }\end{array}$ & $\begin{array}{l}1685 \\
1718\end{array}$ & $\begin{array}{l}935 \\
945\end{array}$ & $\begin{array}{l}303 \\
304\end{array}$ & $\begin{array}{l}924 \\
925\end{array}$ \\
\hline $\mathrm{SiO}_{2}$ & $\begin{array}{l}\text { SPECOMP } \\
\text { Sum }\end{array}$ & $\begin{array}{l}1700 \\
1764\end{array}$ & $\begin{array}{l}938 \\
955\end{array}$ & $\begin{array}{l}305 \\
306\end{array}$ & $\begin{array}{l}944 \\
943\end{array}$ \\
\hline $316 \mathrm{SS}$ & $\begin{array}{l}\text { SPECOMP } \\
\text { Sum }\end{array}$ & $\begin{array}{l}2889 \\
2889\end{array}$ & $\begin{array}{l}743 \\
743\end{array}$ & $\begin{array}{l}198 \\
198\end{array}$ & $\begin{array}{l}481 \\
481\end{array}$ \\
\hline
\end{tabular}




\section{REFERENCES}

1. R. R. Vandervoort, E. L. Raymond, and C. J. Echer, Rddiat, Eff $45(1980)$, 9 )

2. Guide for Irradiation Experiments in EBR-II, Argonne National Laboratory (1975).

3. L. R. Greenwood and L. S. Kellogg, Fusion Reactor Materials Semiannual Progress Report, DOE/ER-0313/12, Neutron Dosimetry for the MOTA-2A Experiment in FFTF (1992) 49-53.

4. L. R. Greenwood, Damage Analysis and Fundamental Studies Quarterly Progress Report, DOE/ER-0046/24 (1986) 5-9.

5. L. R. Greenwood, Recent Research in Neutron Dosimetry and Damage Analysis for Fusion Materials Irradiations, ASTM STP956 (1987) 743-749.

6. D. G. Doran, F. M. Mann, and L. R. Greenwood, Damage Parameters for Candidate Fusion Materials Irradiation Test Facilities, Jou. Nucl. Mater. 174 (1990)125-134.

7. D. R. Davidson, L. R. Greenwood, R. C. Reedy, and W. F. Sommer, Measured Radiation Environment at the C. P. Anderson Los Alamos Meson Physics Facility (LAMPF) Irradiation Facility, ASTM STP870 (1985) 1199-1208.

8. R. C. Birtcher, T. H. Blewitt, M. A. Kirk, T. L. Scott, B. S. Brown, and L. R. Greenwood, Neutron Irradiation Facilities at the Intense Pulsed Neutron Source, Jou. Nucl. Mater. 108\&109 (1982) 3-9.

9. S. Berg and W. N. McElroy, A Computer-Automated Iterative Method for Neutron Flux Spectra Determination by Foil Activation, AFWL-TR-67-41, Atomics International (1967).

10. F. Schmittroth, FERRET Data Analysis Code, HEDL-TME 79-10, Hanford Engineering Development Laboratory (1979).

11. F. G. Perey, Least Squares Dosimetry Unfolding: The Program STAY'SL, ORNL/TM-6062, Oak Ridge National Laboratory (1977).

12. Evaluated Nuclear Data File, National Nuclear Data Center, Brookhaven National Laboratory.

13. E. P. Lippinott and W. N. McElroy, Power Reactor Benchmark Studies, ASTM STP1001 (1989) 308-313.

14. L. R. Greenwood, R. R. Heinrich, R. J. Kennerley, and R. Medrzychowski, Nucl. Technol. 41 (1978) 109. 
15. L. R. Greenwood, R. R. Heinrich, M. J. Saltmarsh, and C. B. Fulmer, Nucl. Sci. Eng. 72 (1979) 175.

16. F. M. Mann, D. E. Lessor, and J. S. Pintler, REAC Nuclear Data Libraries, Radiation Effects 92-96 (1985) 207-210.

17. F. M. Mann, Transmutation of Alloys in MFE Facilities as Calculated by REAC, HEDL-TME 81-37, Hanford Engineering Development Laboratory (1982).

18. G. J. Butterworth and L. Giancarli, Some Radiological Limitations on the Compositions of Low-Activation Materials for Power Reactors, Jou. Nucl. Mater. 155157 (1988) 575-580.

19. S. Cierjacks, Low-Activation Fusion Materials Development and Related Nuclear Data Needs, IAEA-TECDOC-572 (1989) 53-64.

20. P. J. Maziasz, Effects of Helium Content on Microstructural Development in Type 316 Stainless Steel Under Neutron Irradiation, ORNL-6121, Oak Ridge National Laboratory (1985).

21. L. R. Greenwood, D. W. Kneff, R. P. Skowronski, and F. M. Mann, Jou. Nucl. Mater. 122-123 (1984) 1002

22. L. R. Greenwood, A New Calculation of Thermal Neutron Damage and Helium Production in Nickel, Jou. Nucl. Mater. 116 (1983)137-142.

23. A. W. Longest, J. E. Corum, D. E. Heatherly, and K. R. Thoms, Design of Spectrally Tailored Fusion Reactor Materials Experiments in the HFIR RB* Capsule Irradiation Facility, Jou. Nucl. Mater. 155-157 (1988) 1346-1349.

24. L. R. Greenwood, F. A. Garner, and B. M. Oliver, Helium Generation Rates in Isotopicaliy Tailored Fe-Cr-Ni Alloys Irradiated in FFTF/MOTA, Jou. Nucl. Mater. 191194 (1992) 1051-1055.

25. L. K. Mansur and M. L. Grossbeck, Mechanical Property Changes Induced in Structural Alloys by Neutron Irradiations with Different Helium to Displacement Ratios, Jou. Nucl. Mater. 155-157 (1988) 130-147.

26. D. L. Smith, H. Matsui, L. Greenwood, and B. Loomis, Jou. Nucl. Mater. 155-157 (1988) 1359-1363

27. D. W. Kneff, L. R. Greenwood, B. M. Oliver, and R. P. Skowronski, Radiation Effects $93(1986) 217$

28. L. R. Greenwood, D. G. Graczyk, and D. W. Kneff, Jou. Nucl. Mater. 155-157 (1988) 1335-1339 
29. L. R. Greenwood and F. A. Garner, Transmtation of Mo, Re, W, Nb, Hf, and $\mathrm{Zr}$ in Various Irradiation Test Facilities and STARFIRE, Proceedings of the Int. Conf. on Fusion Reactor Materials, Stressa, Italy, Sept. 27-Oct. 1, 1993.

30. L. R. Greenwood and R. K. Smither, SPECTER: Neutron Damage Calculations for Materials Irradiations, ANL/FPP-TM-197 (1985).

31. J. Lindhard, V. Nielsen, M. Scharff, and P. V. Thomsen, Mat. Phys. Medd. Dar. V. D. Selk, 22 (1963) 10.

32. G. R. Odette and D. R. Dorian, Nucl. Technol. 29 (1976) 346.

33. R. E. Macfarlane, D. W. Muir, and F. M. Mann, Jou. Nucl. Mater. 122-123 (1984) 1041.

34. L. R. Greenwood, SPECOMP Calculations of Radiation Damage in Compounds, ASTM STP1001 (1989) 598-602.

35. L. R. Greenwood, Radiation Damage Calculations for Compound Materials, ASTMSTP1046 (1990) 633-641.

36. H. L. Heinisch, J. Nucl. Mater. 178 (1991) 19.

37. H. Wiedersich, J. Nucl. Mater. 179-181 (1991) 70.

38. STARFIRE: A Commercial Tokamak Fusion Power Plant Study, ANL/FPP-80-1 (1980). 
Figure Captions

1. Comparision of three materials effects in 316 stainless steel following irradiations in three radically different facilities. Note that whereas little correlation exists on the basis of neutron exposure (flux $>1 \mathrm{MeV}$ ), the effects correlate quite nicely on the basis of dpa.

2. The neutron spectrum is shown for the fission of ${ }^{235} \mathrm{U}$. The spectrum is welldescribed by a Maxwellian with a nuclear temperature of about $1.5 \mathrm{MeV}$.

3. Comparison of neutron spectra for the mixed-spectrum reactor HFIR and the fast reactor FFTF. Both spectra are at midplane at current reduced power levels.

4. The HFIR neutron spectrum is plotted in three different representations: differential point spectrum (solid line), group flux (dashed line), group flux times energy (flux/lethargy)(dotted line).

5. Neutron spectra as a function of angle from the incident deuterium beam direction at the $14 \mathrm{MeV}$ neutron source RTNSII.

6. Neutron spectra for various accelerator-based neutron sources proposed to simulate fusion reactor spectra, including a fusion first wall spectrum (DEMO), a fusion beam plasma device, a $\operatorname{Li}(d, n)$ accelerator neutron source, two spallation neutron sources the European Accelerator (EURAC), and a $H(t, n)$ water-tritium accelerator neutron source.

7. Neutron spectra at the LASREF and IPNS spallation neutron sources, showing both calculations and spectral adjustment with the STAY'SL. computer code.

8. The energy dependence of several neutron activation cross sections is illustrated for (a) the thermal $(n, y)$ reactions on $A u$ and $C o$ and (b) fast threshold reactions.

9. The energy dependence of various neutron activation reactions is illustrated for the HFIR mixed-spectrum fission reactor. The horizontal bar by each reaction indicates the energy region where $90 \%$ of the activity is generated.

10. Neutron spectral adjustment is illustrated at in-core and below-core positions in the Fast Flux Test Facility. The solid lines show the neutronics calculations and the dotted lines the result of spectral adjustments with STAY'SL.

11. The production of helium is shown as a function of total neutron fluence in HFIR for the ${ }^{58} \mathrm{Ni}(\mathrm{n}, y)^{59} \mathrm{Ni}(\mathrm{n}, a)^{56} \mathrm{Fe}$ reaction. The solid circles represent the experimental 
data and the open circles are calculations based on neutron dosimetry and recommended cross sections.

12. Helium production from copper is shown as a function of thermal neutron fluence. The squares are measured values, and the dotted line represents calculations. The dashed line shows the helium production from $\mathrm{Cu}(n, a)$ fast neutron reactions.

13. Transmutation of tungsten in the mixed-spectrum reactor HFIR and the fusion reactor STARFIRE. Note the rapid transmutation to rhenium followed by the transmutation of rhenium to osmium.

14. Energy distribution of nickel recoils in HFIR. Each reaction is shown separately and labelled by the outgoing particle. Note that elastic and inelastic produce most of the recoils at lower energies; however, $(n, a)$ reactions produce recoils up to $\mathrm{MeV}$ energies.

15. Integral recoil spectra are illustrated for various irradiation facilities including FFTF, HFIR, IPNS, a pure fission spectrum, a $14 \mathrm{MeV}$ spectrum, and a fusion first-wall spectrum.

16. Displacement cross sections are shown for nickel as a function of neutron energy. The contributions are shown from elastic scattering $(n)$, inelastic scattering $\left(n^{\prime}\right)$, proton emission $(p)$, alpha emission $(a)$, and the $(n, 2 n)$ reaction.

17. Displacement damage cross sections are illustrated for the compound $\mathrm{Li}_{2} \mathrm{O}$ showing each combination of recoil atom and matrix atom.

18. Displacement damage cross sections for the compound $\mathrm{Li}_{2} \mathrm{O}$ are compared with the sum of $2 \mathrm{Li}+0$. Note that the correct compound damage from SPECOMP is considerably higher than would be obtained from a simple sum of the elements.

19. Comparison of the defect survivability models Hmod and Wmod with the standard dpa model. Note that both models predict that low energy recoils produce more stable defects than higher energy recoils; however, little energy dependence exists above about $2 \mathrm{keV}$.

20. Modified damage cross sections using the Hmod and Wmod models of defect survivability are compared with the standard dpa cross section for iron. Note that both models lead to less net damage; however, relatively more damage occurs at lower neutron energies.

21. Comparison of different irradiation facilities on the basis of displacement damage vs. helium production. The fusion power reactor spectrum is used as a guide to assess possible sources for fusion materials studies. 


\section{HIGH-ENERGY NEUTRON IRRADIATION EFFECTS ON THE TENSILE PROPERTIES AND MICROSTRUCTURE OF 316 \\ STAINLESS STEEL $†$}

R. R. VANDER VOORT, E. L. RAYMOND and C. J. ECHER

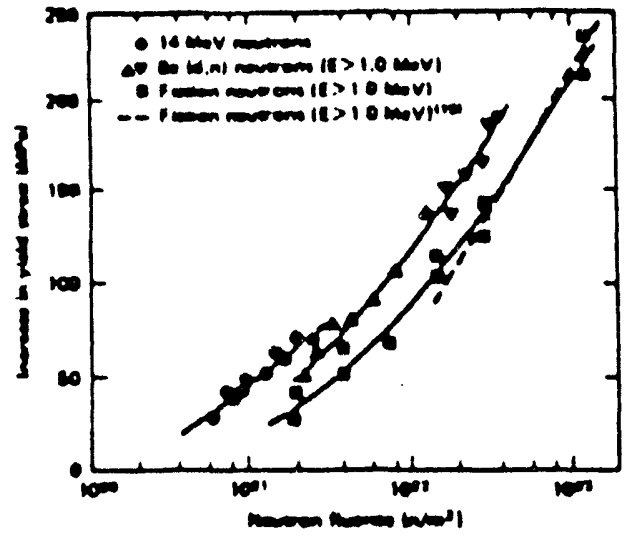

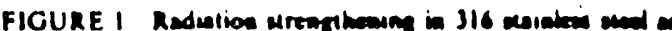
- hunction of exwion furmos.

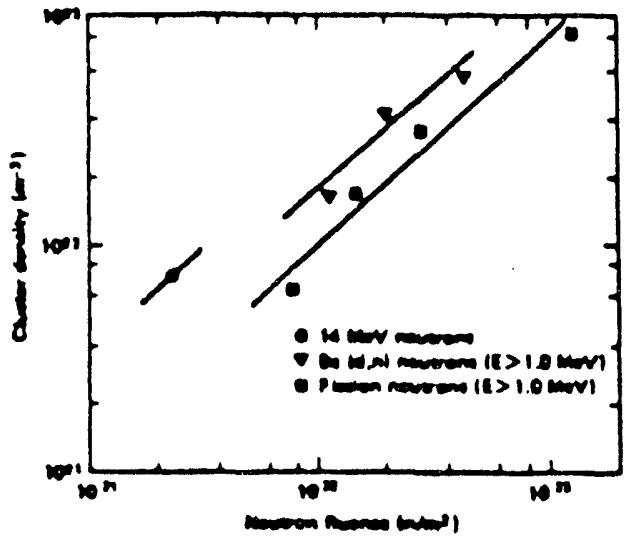

FIGURF. 5 Drming of redution monerd defects in 316

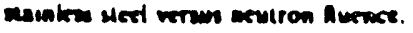

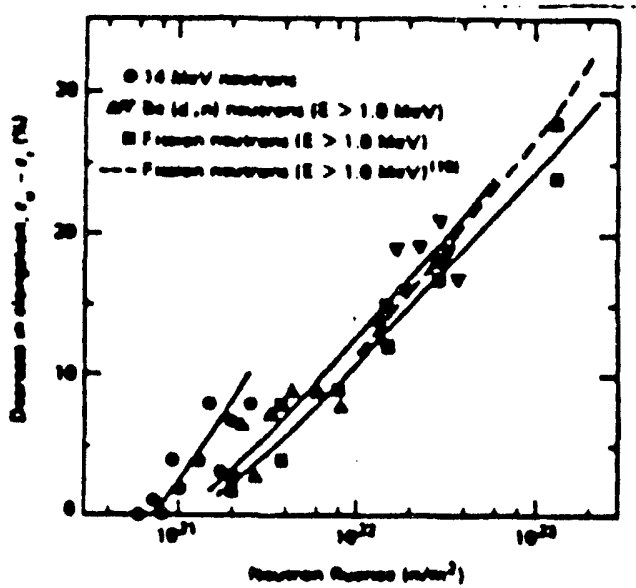

FIGURE 3 Derrease in ductingy of mouron intedinced 316

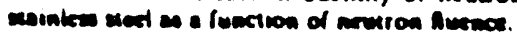

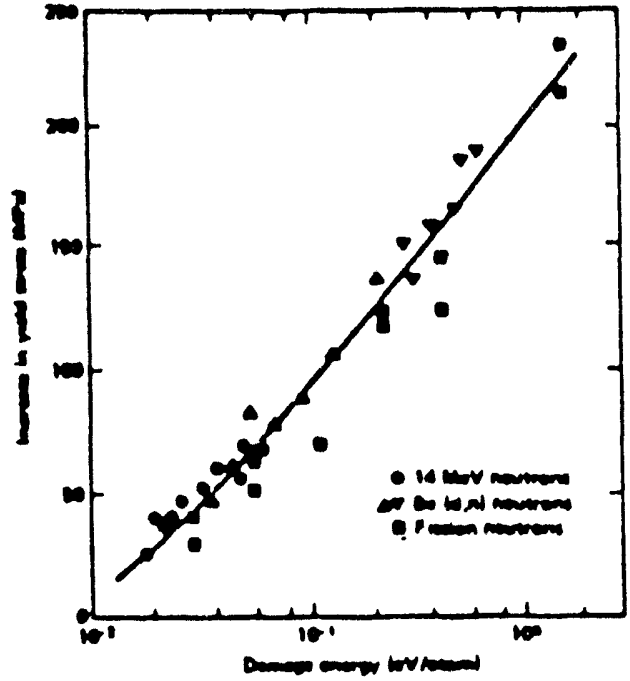

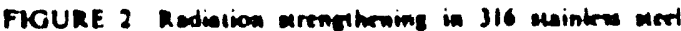
verum demsere energy.

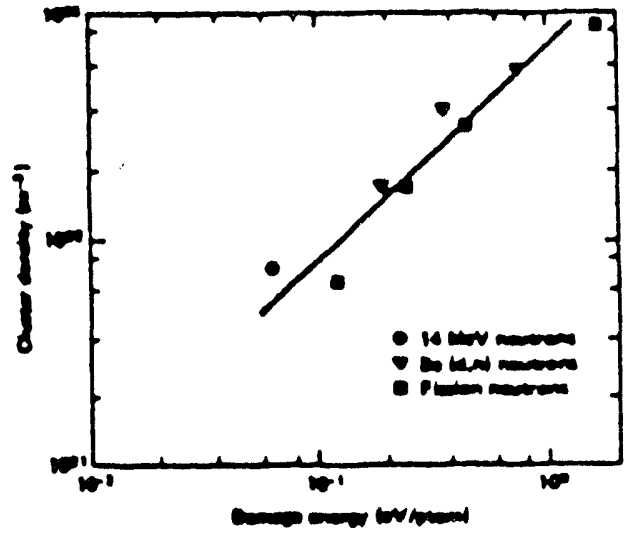

FHOURE - Daming of matiotion indeced dofocte in 316

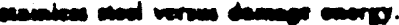

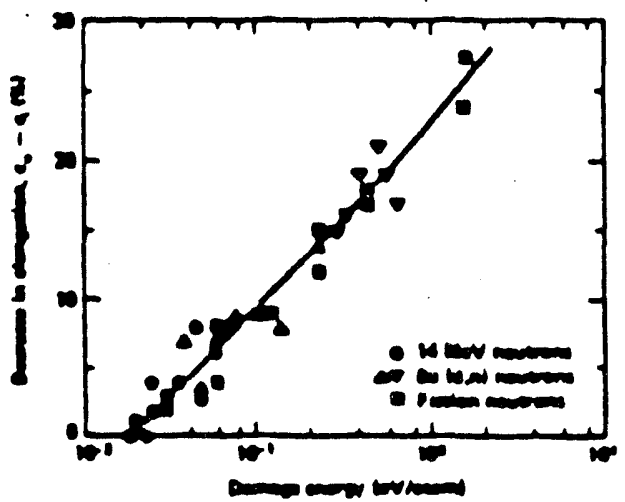

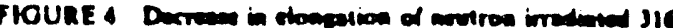

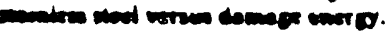




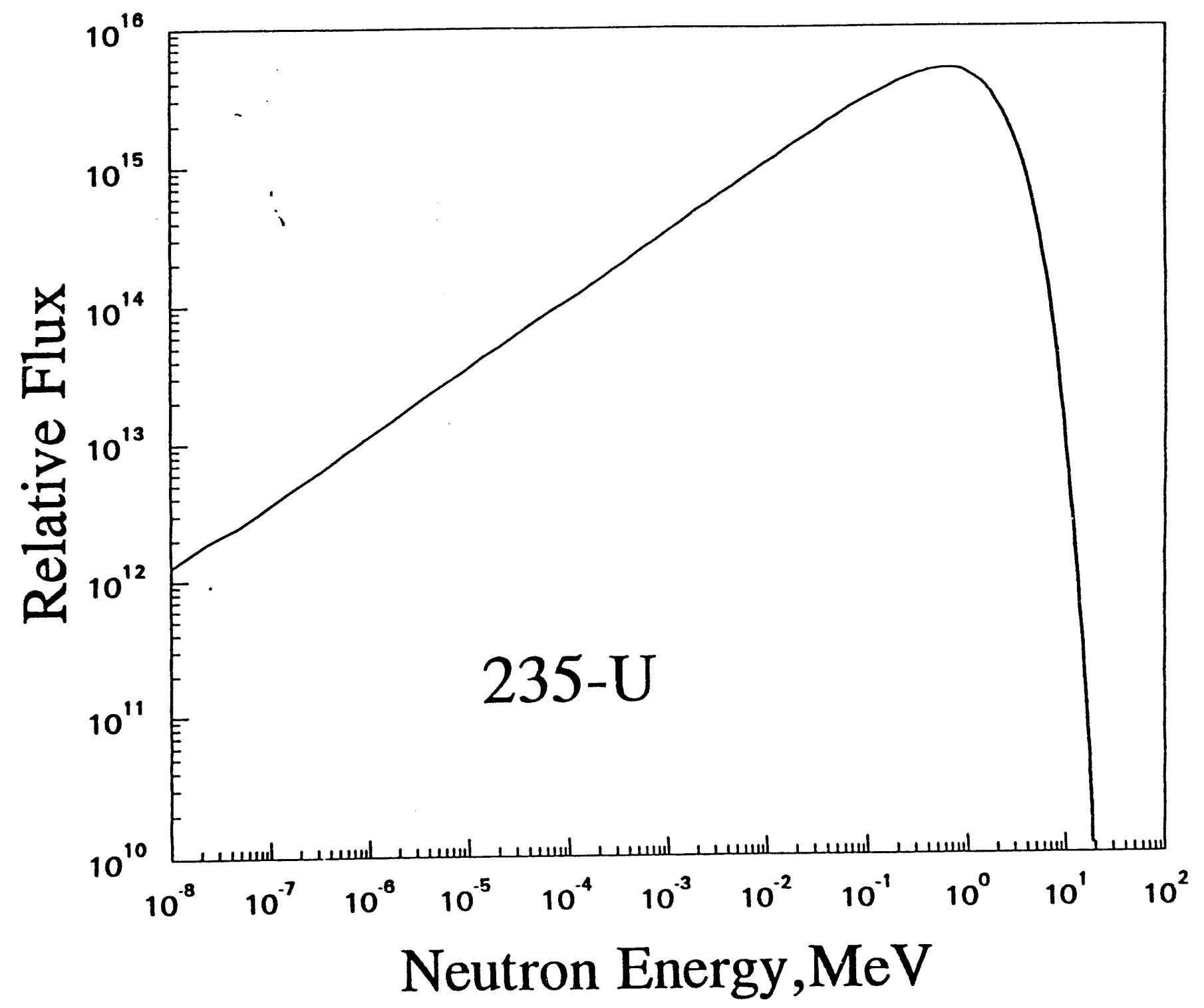




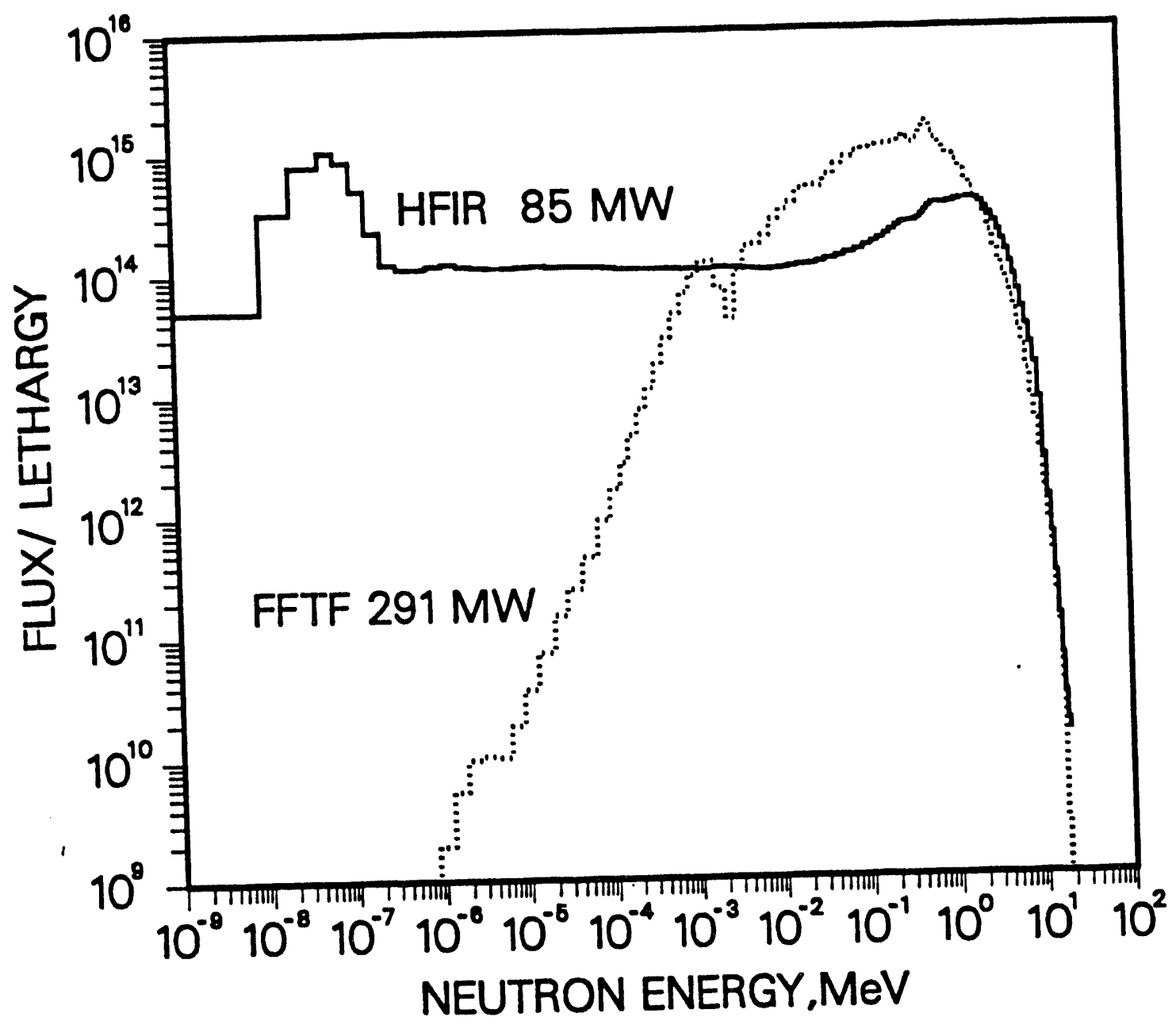




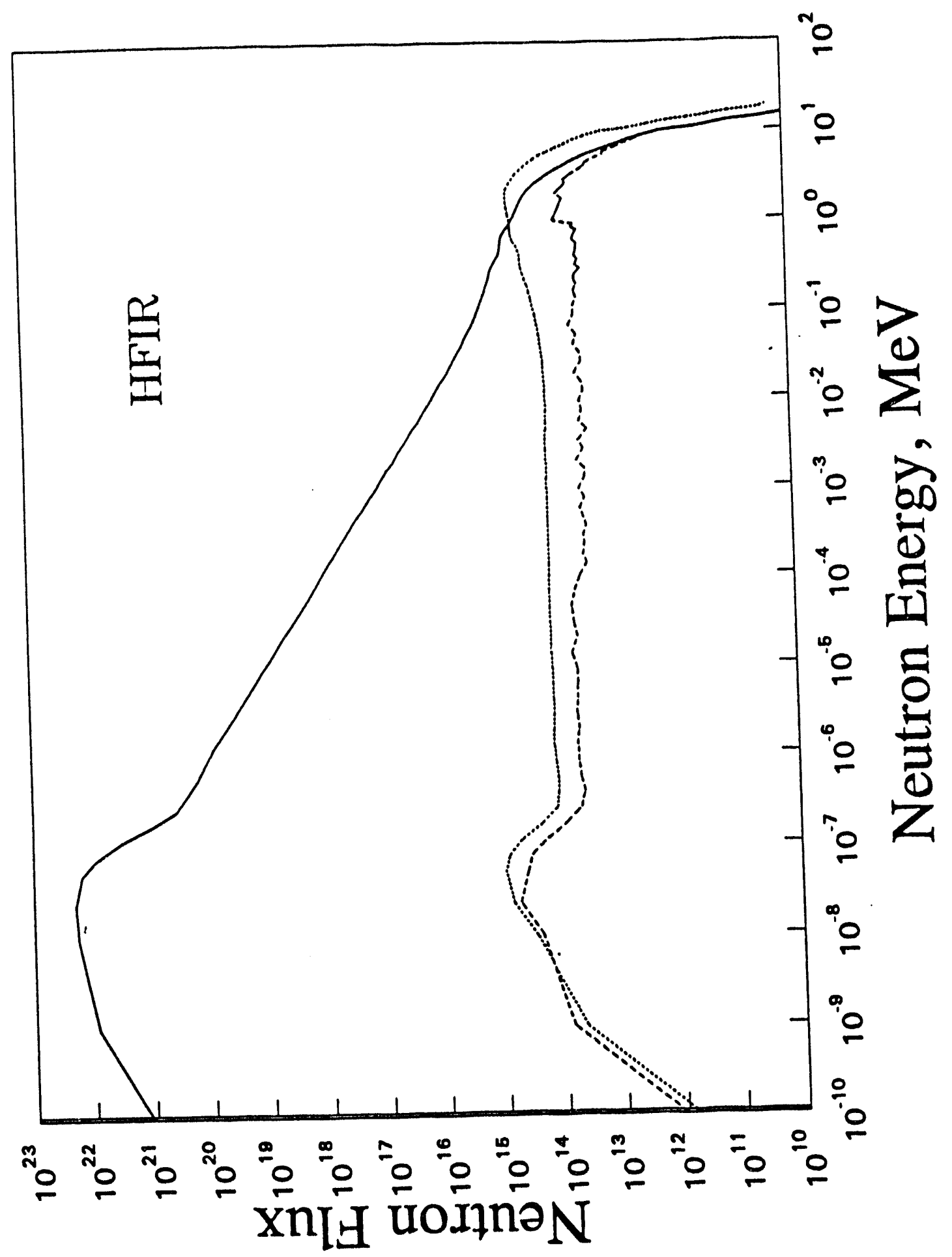




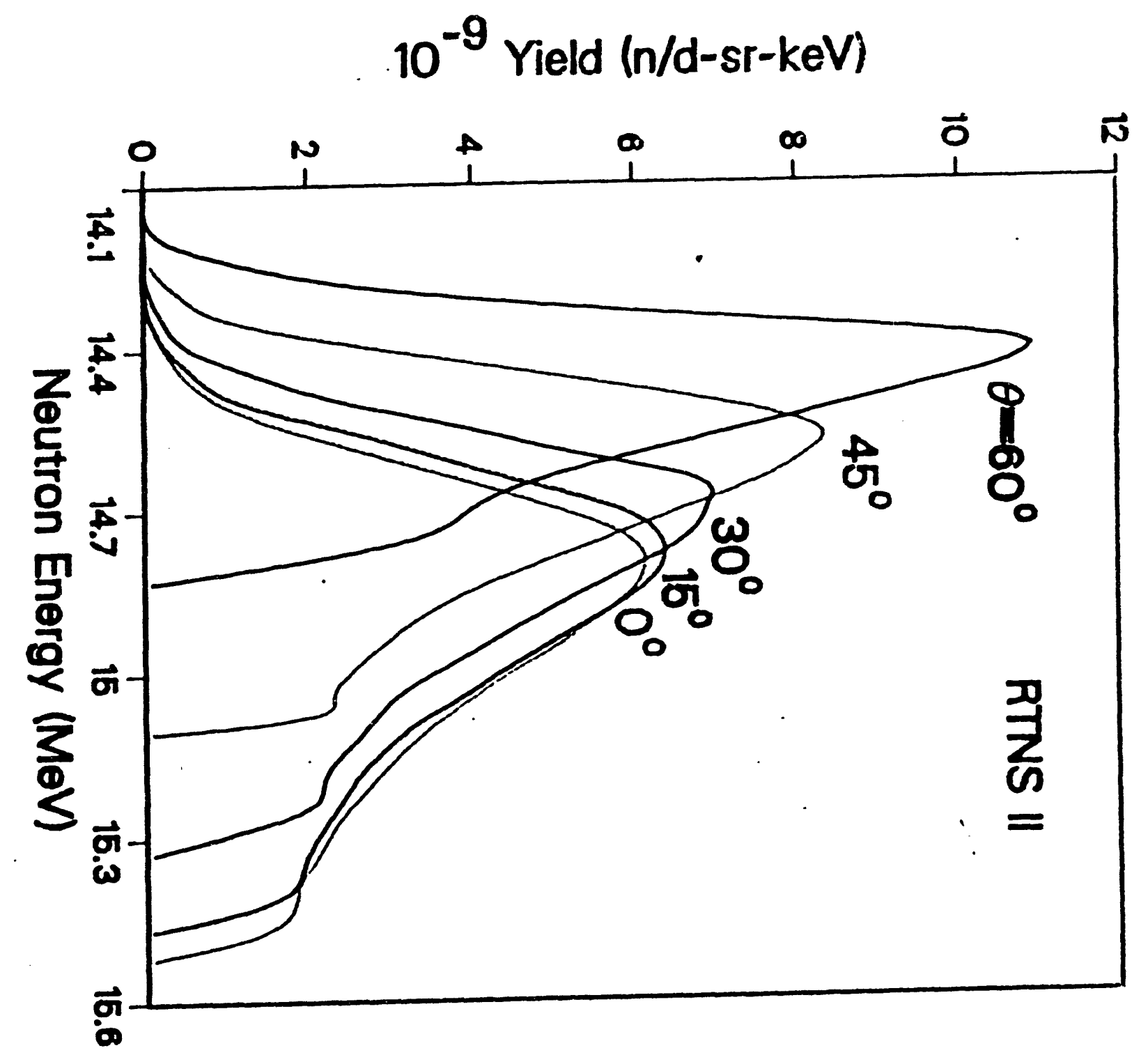




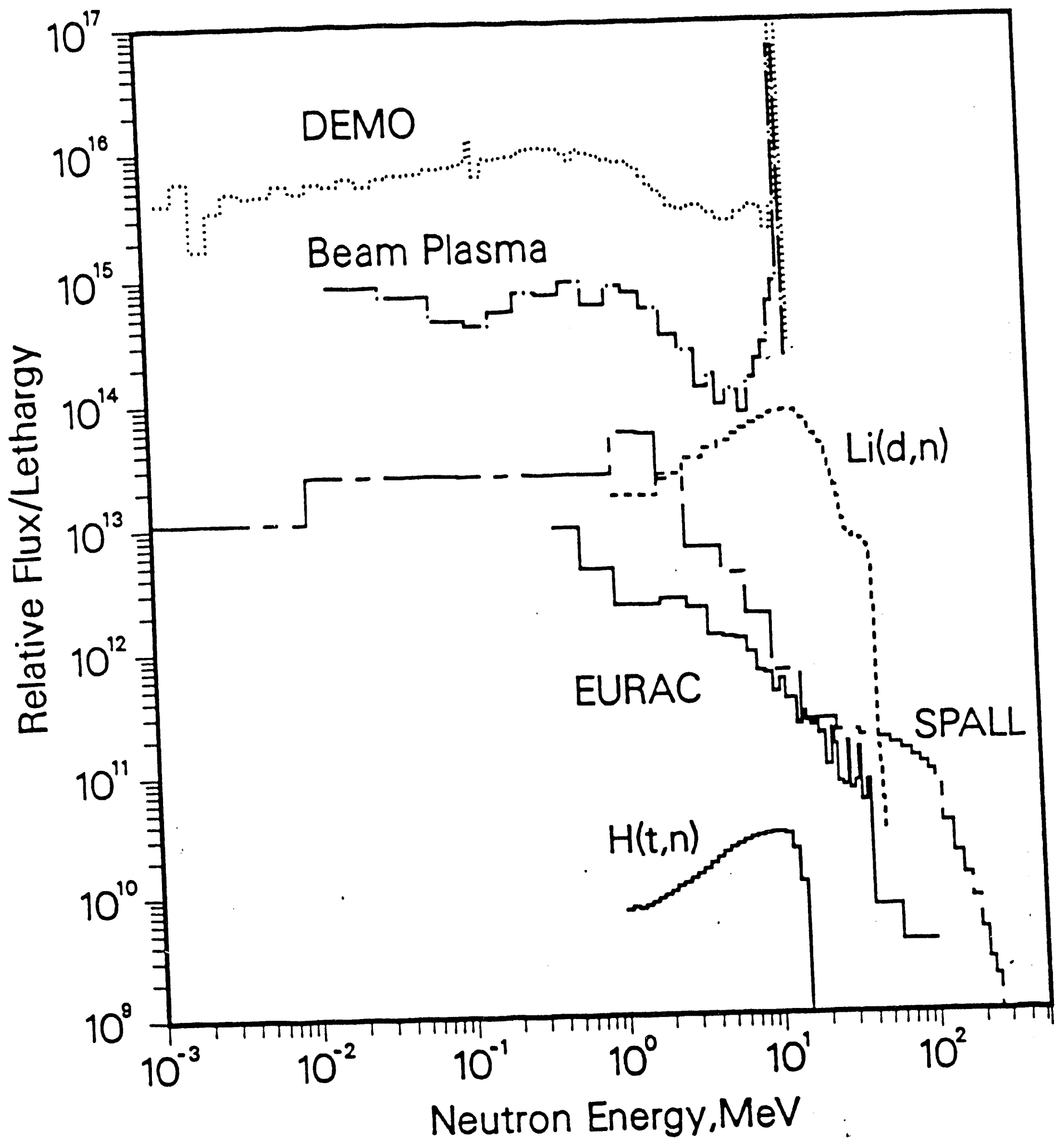




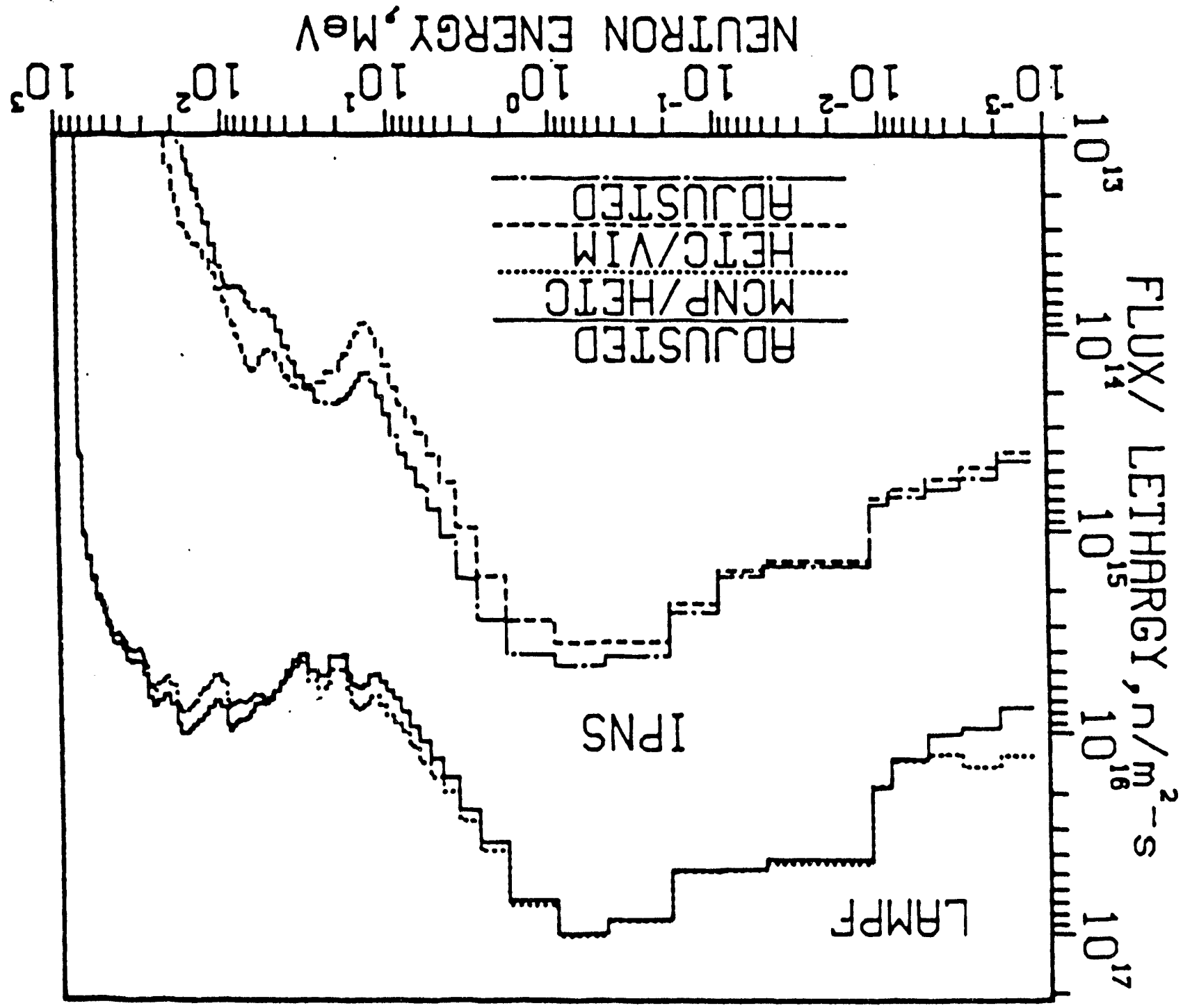




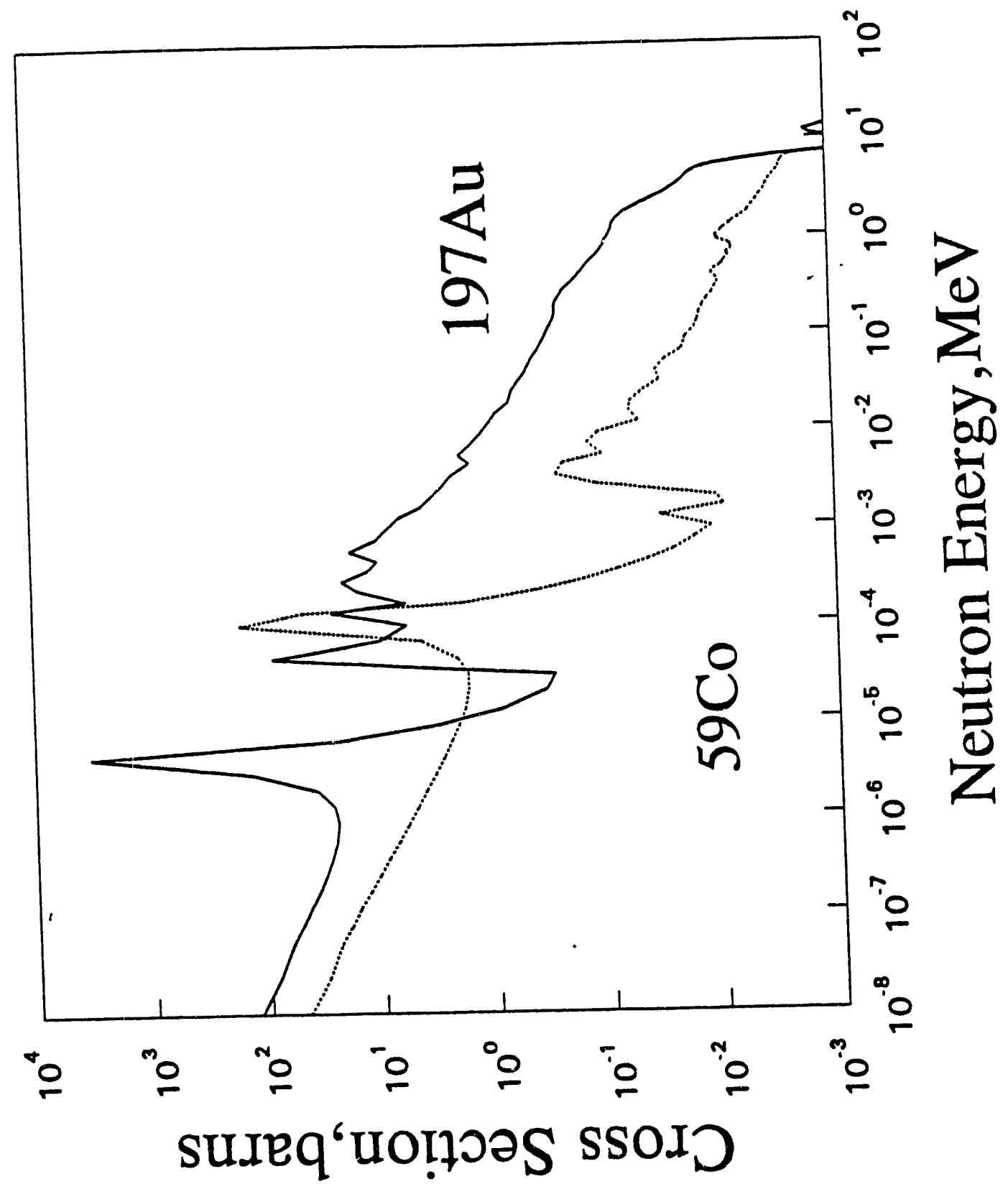




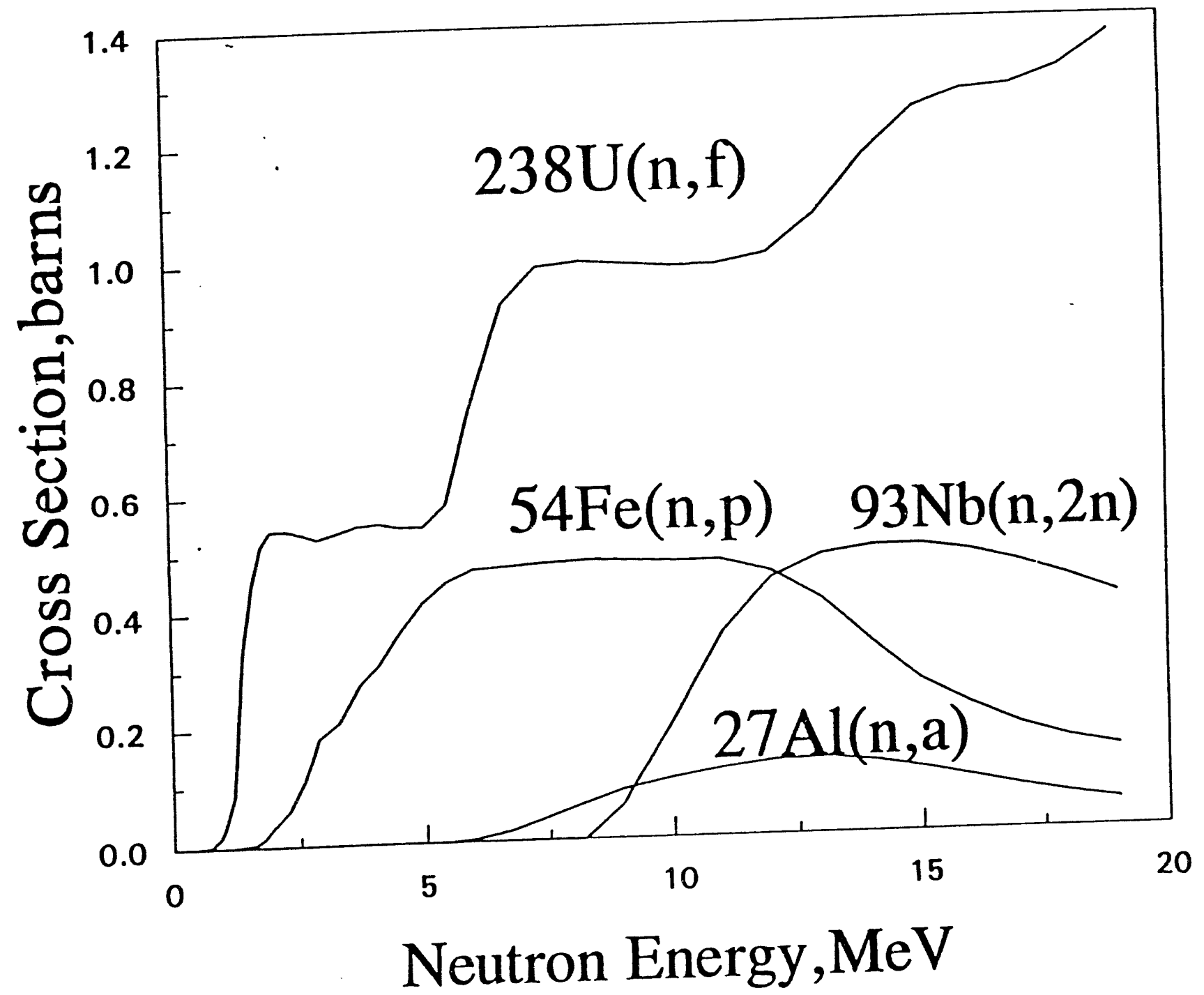

$\infty$ 


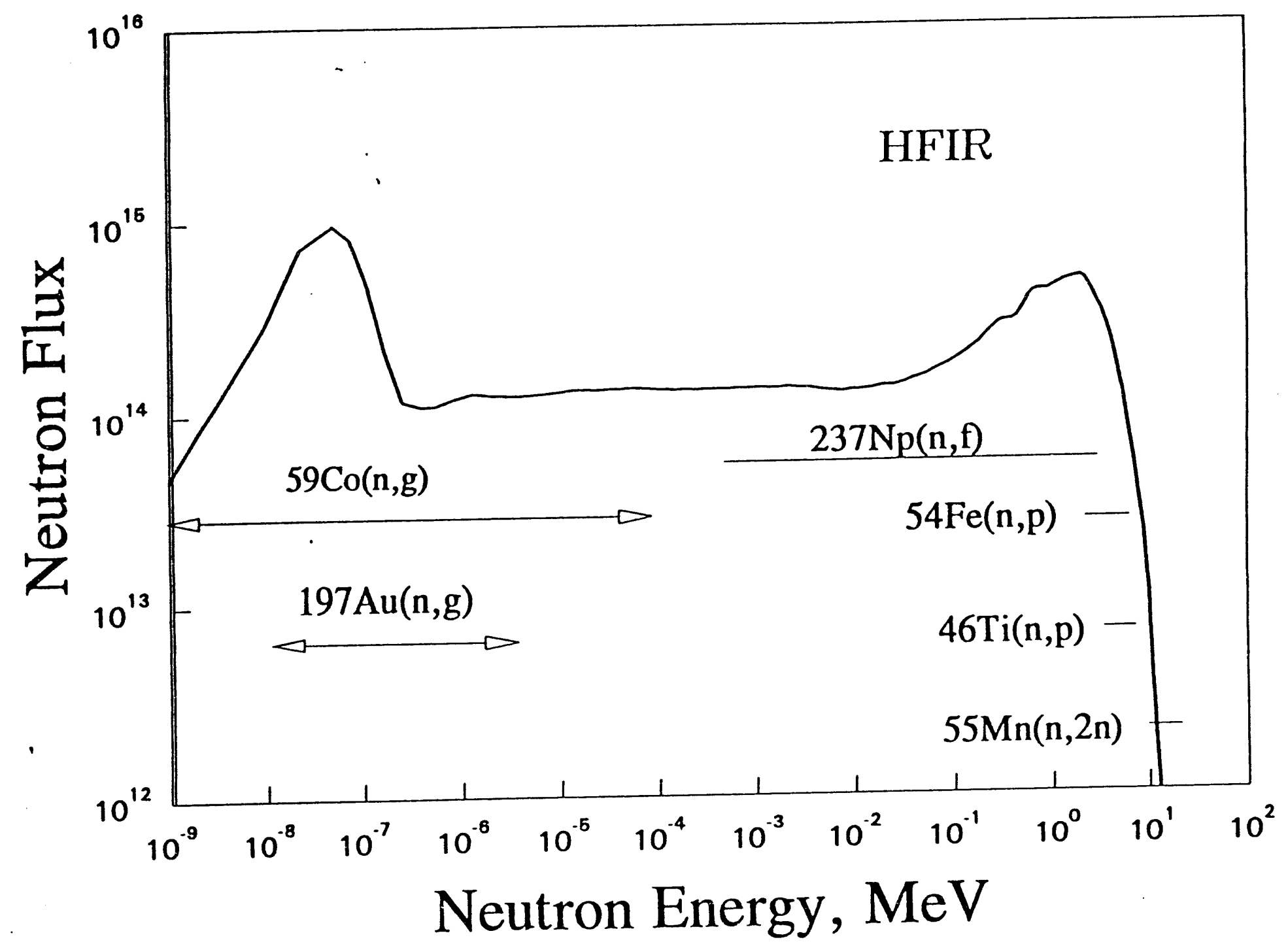




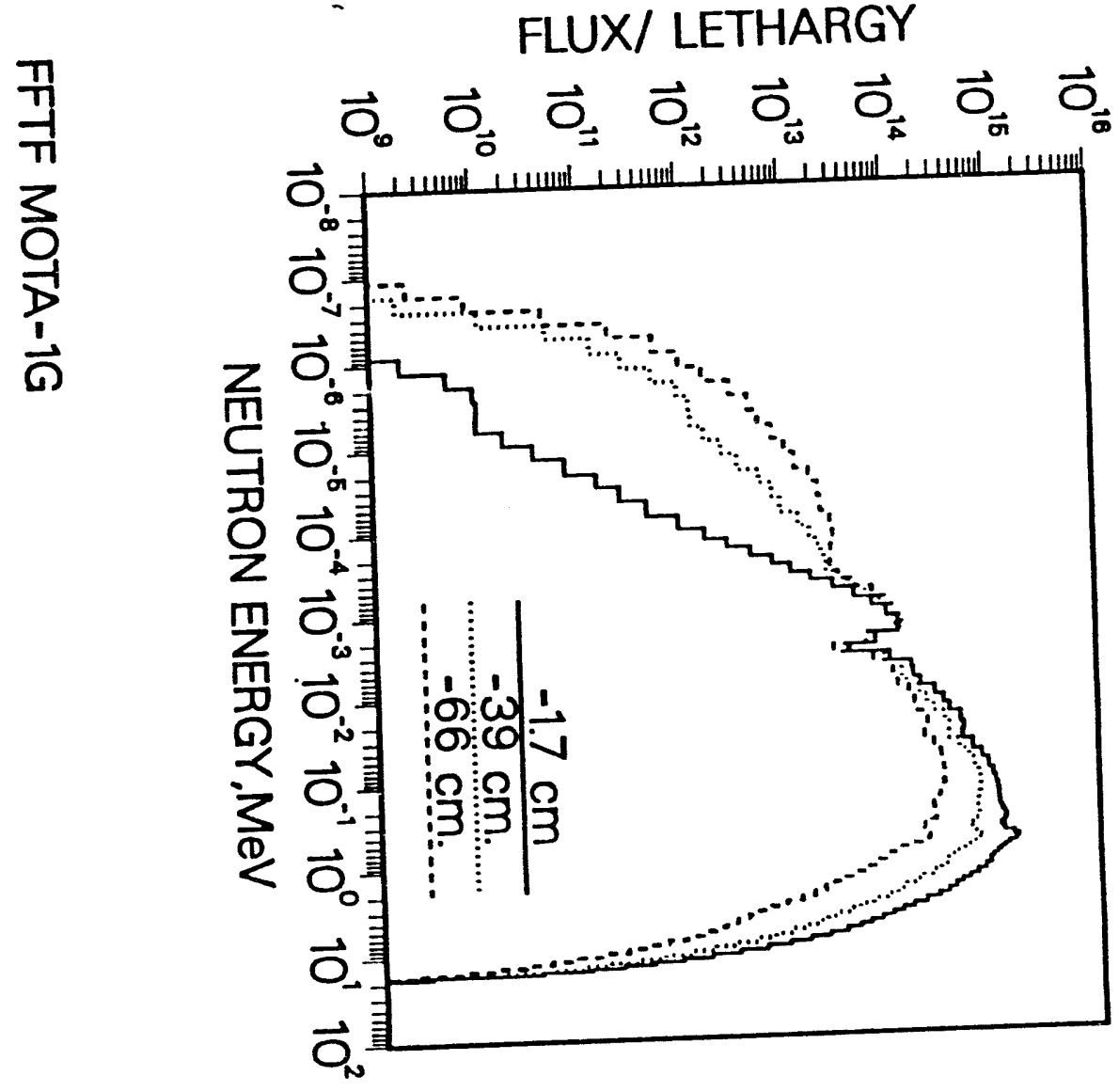




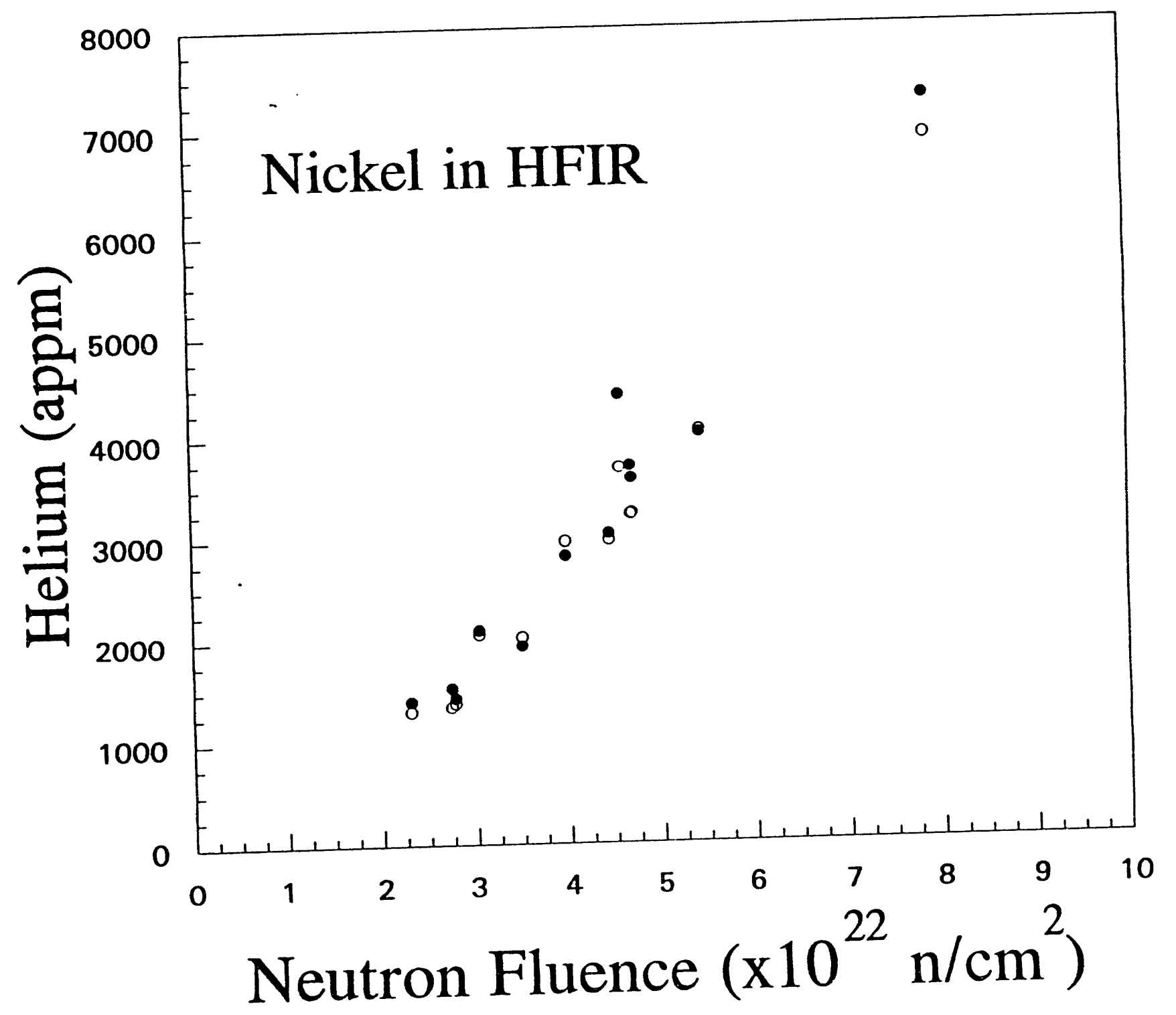


$\Lambda$

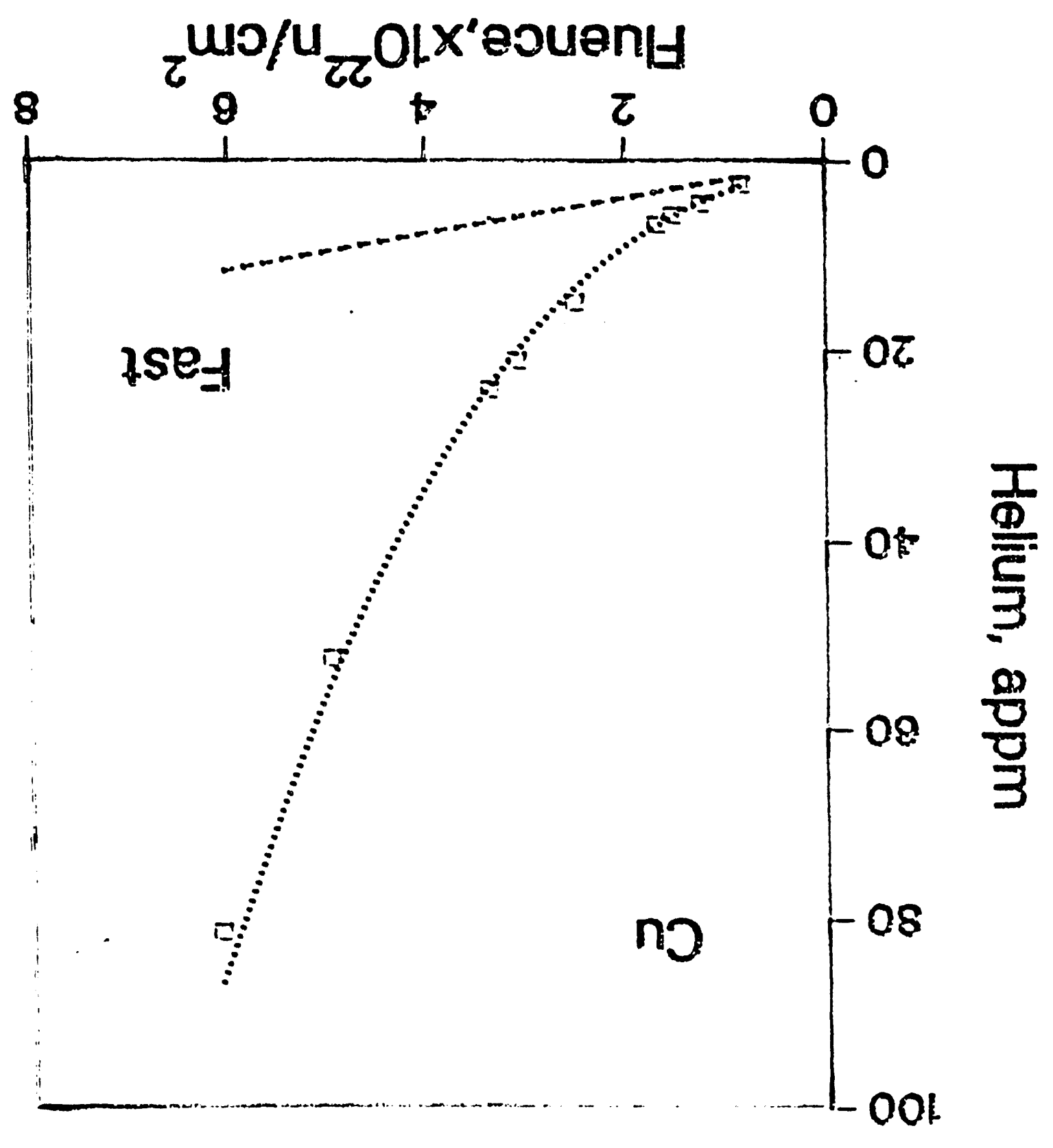



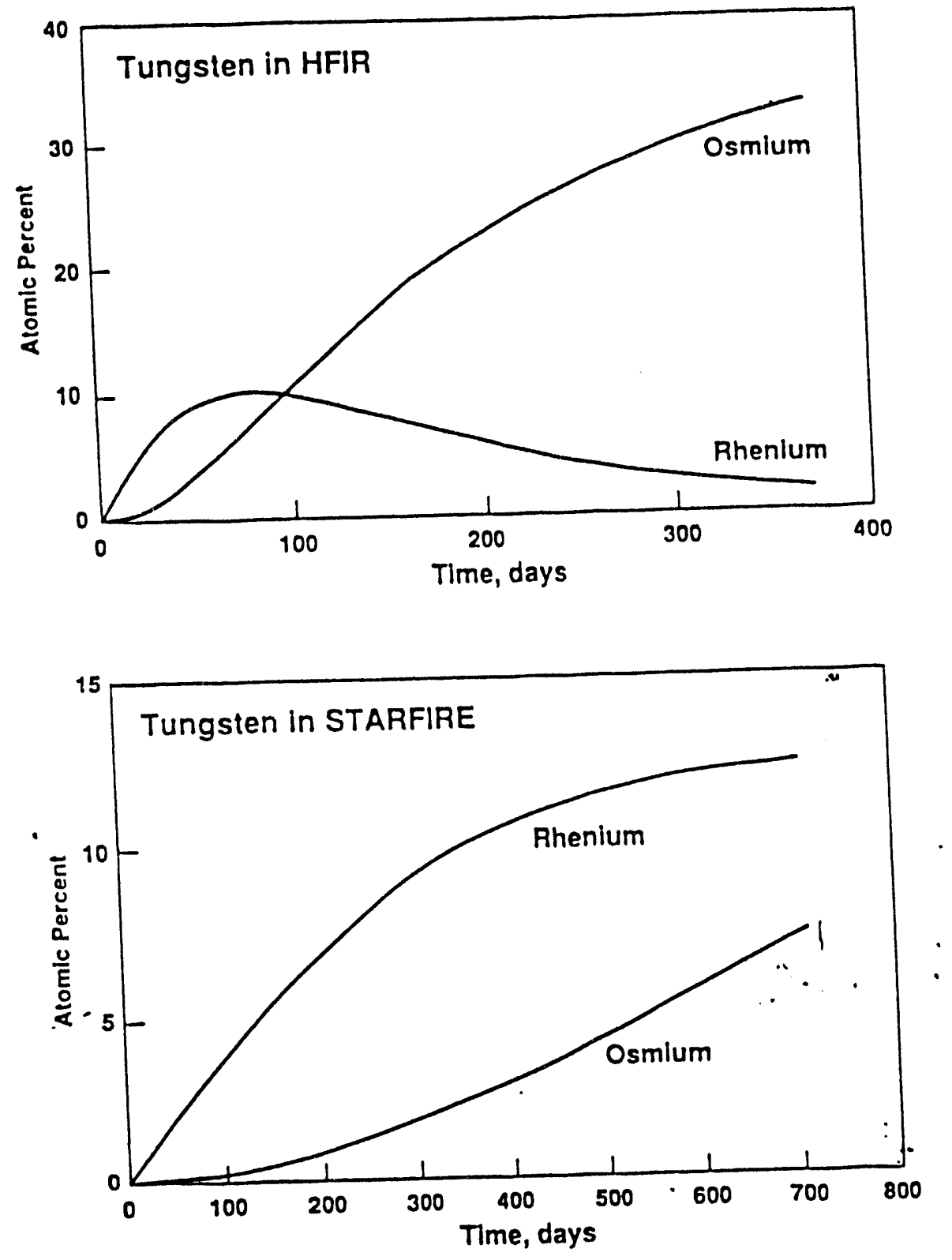

18. Predicted formation of rhenium and osmium irradiated in (a) the PTP position of HFIR at $85 \mathrm{MW}$, and (b) the first wall position of STARFIRE at $3.8 \mathrm{MW} / \mathrm{m}^{2}$. (59) Note that the relative amounts of osmium and rhenium are reversed in the two reactors. 


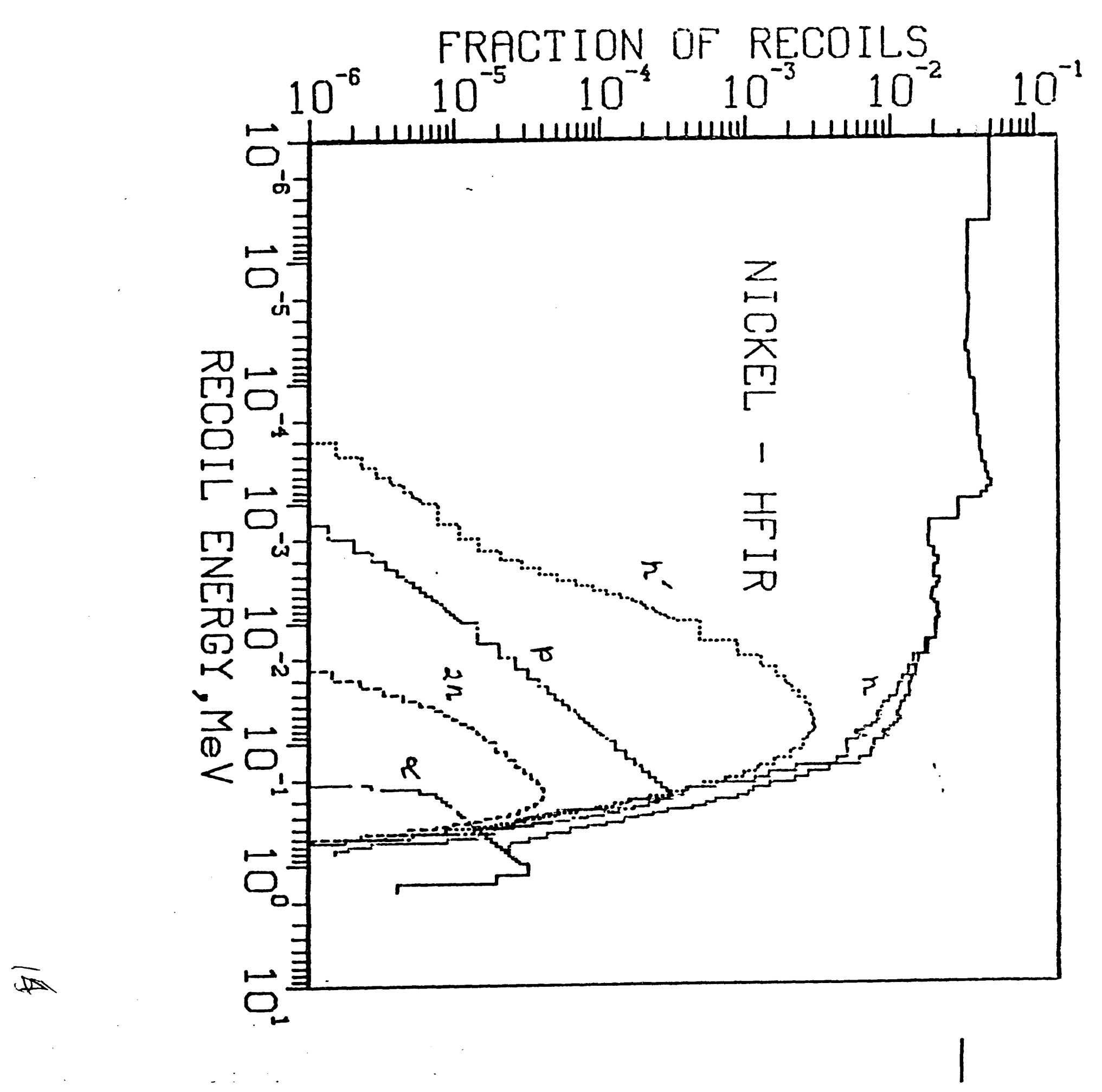




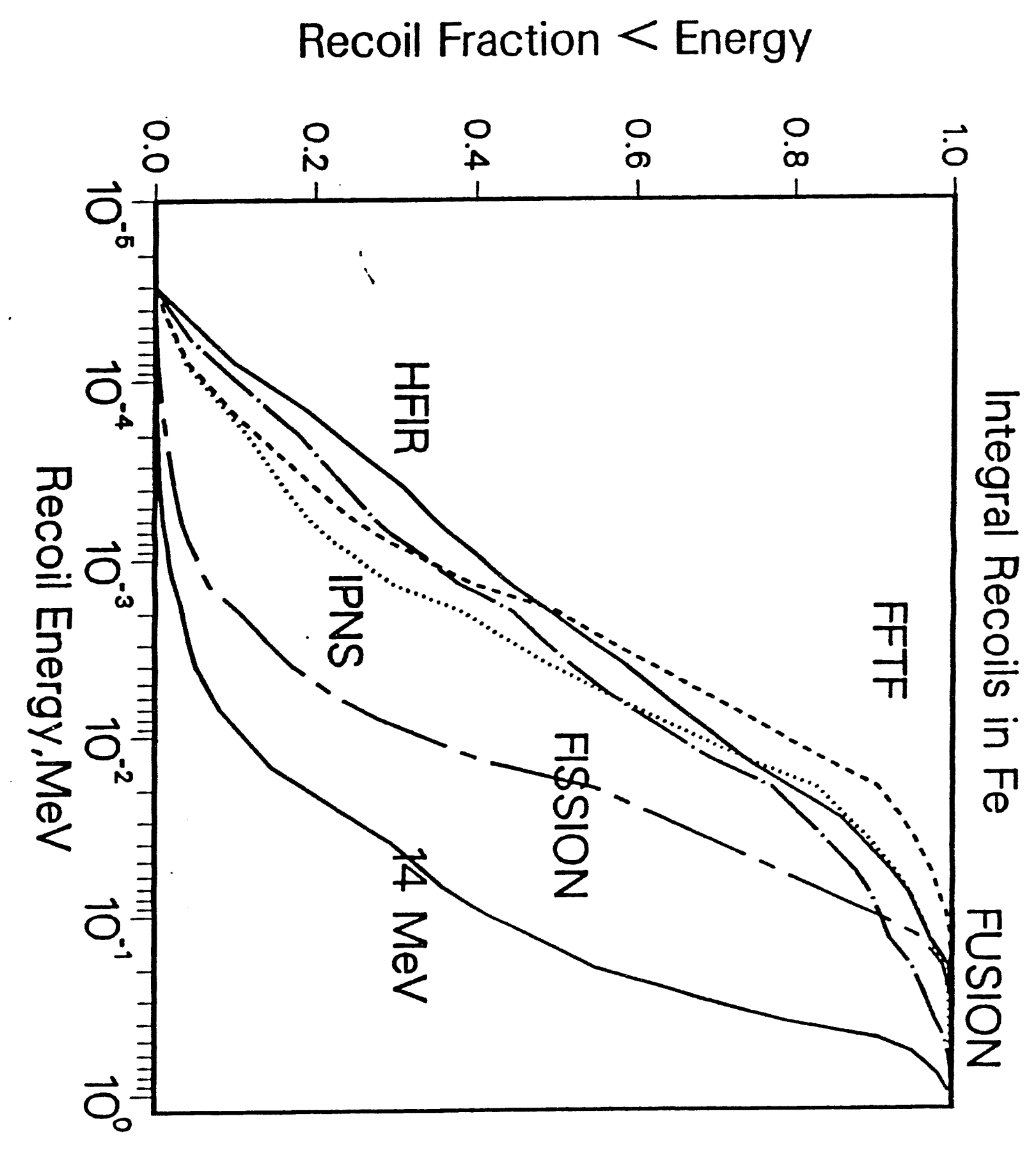

5 


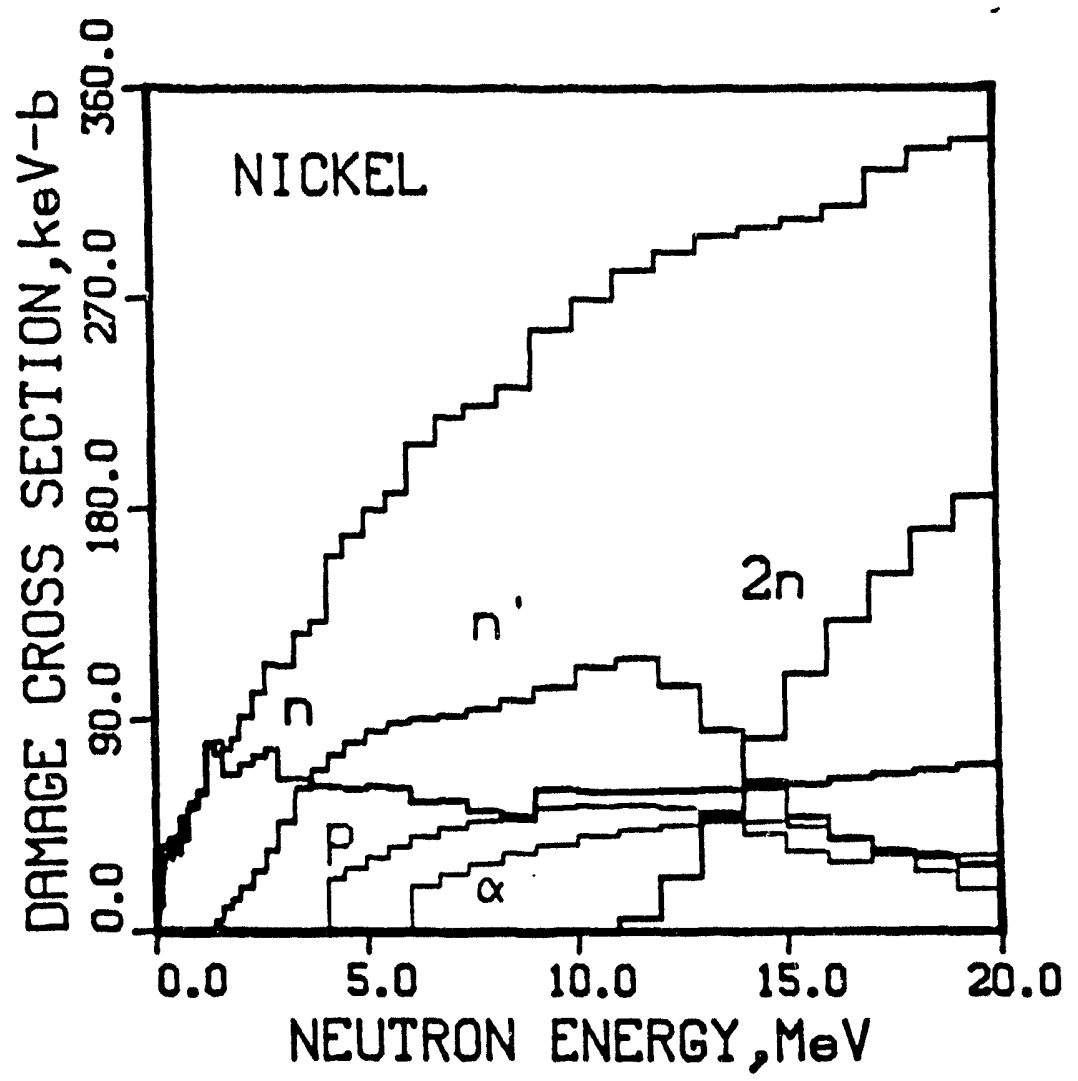




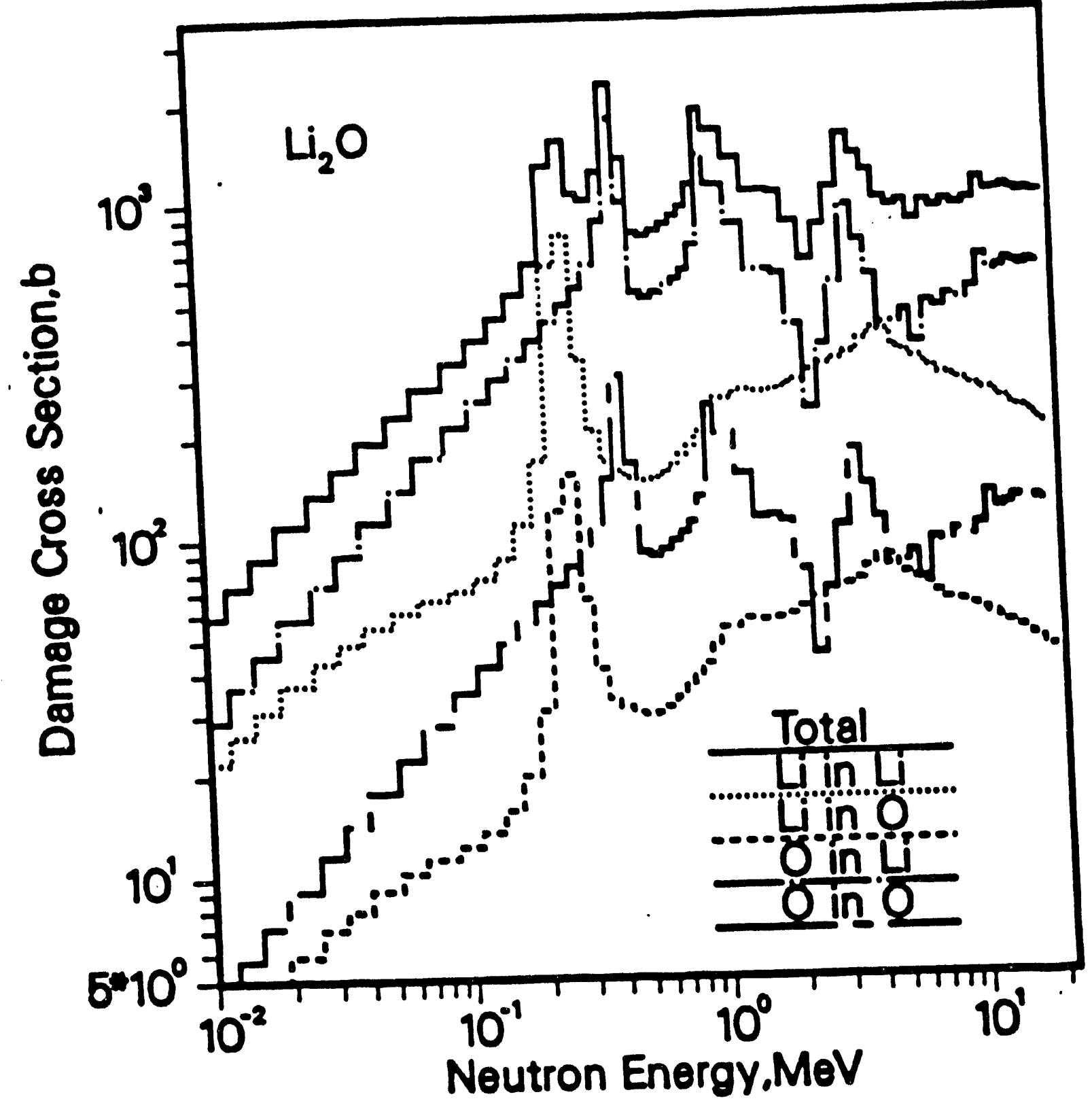


SPECOMP computer code: dpa cross sections for compounds. comparison of SPECOMP and elemental sum from SPECTER predicts increased damage in tritium breeder materials; same damage rates in structural materials.

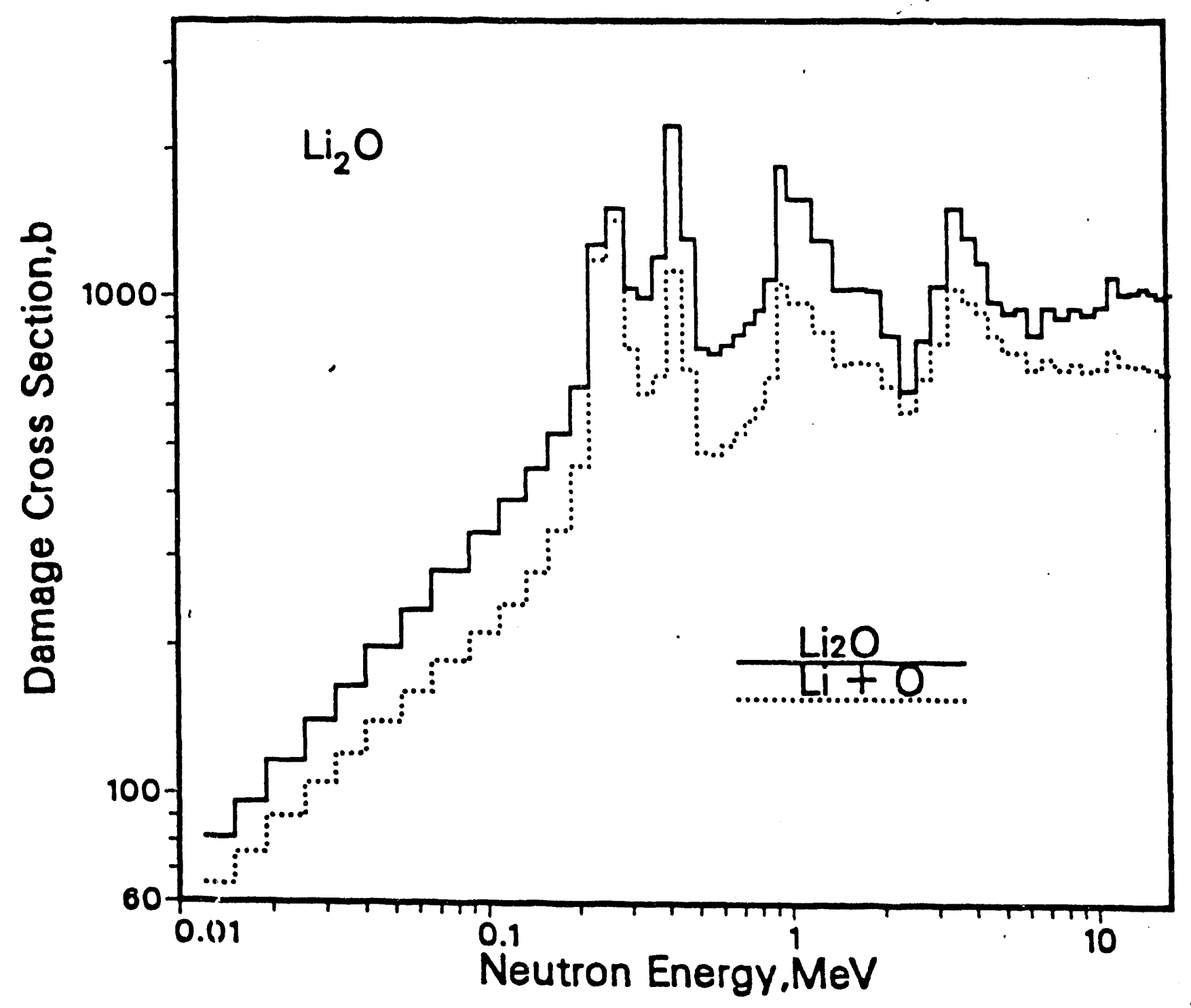




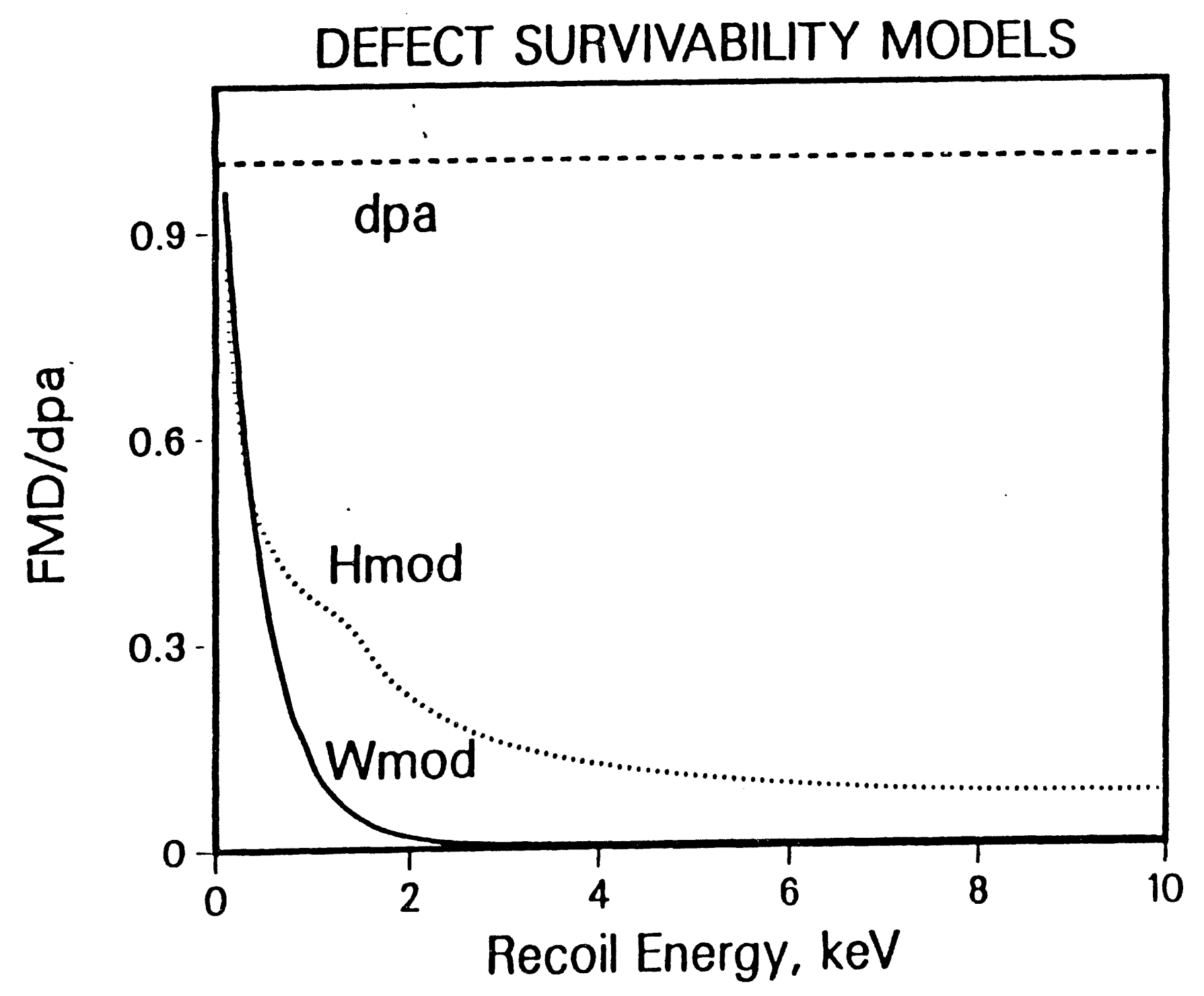

兽 


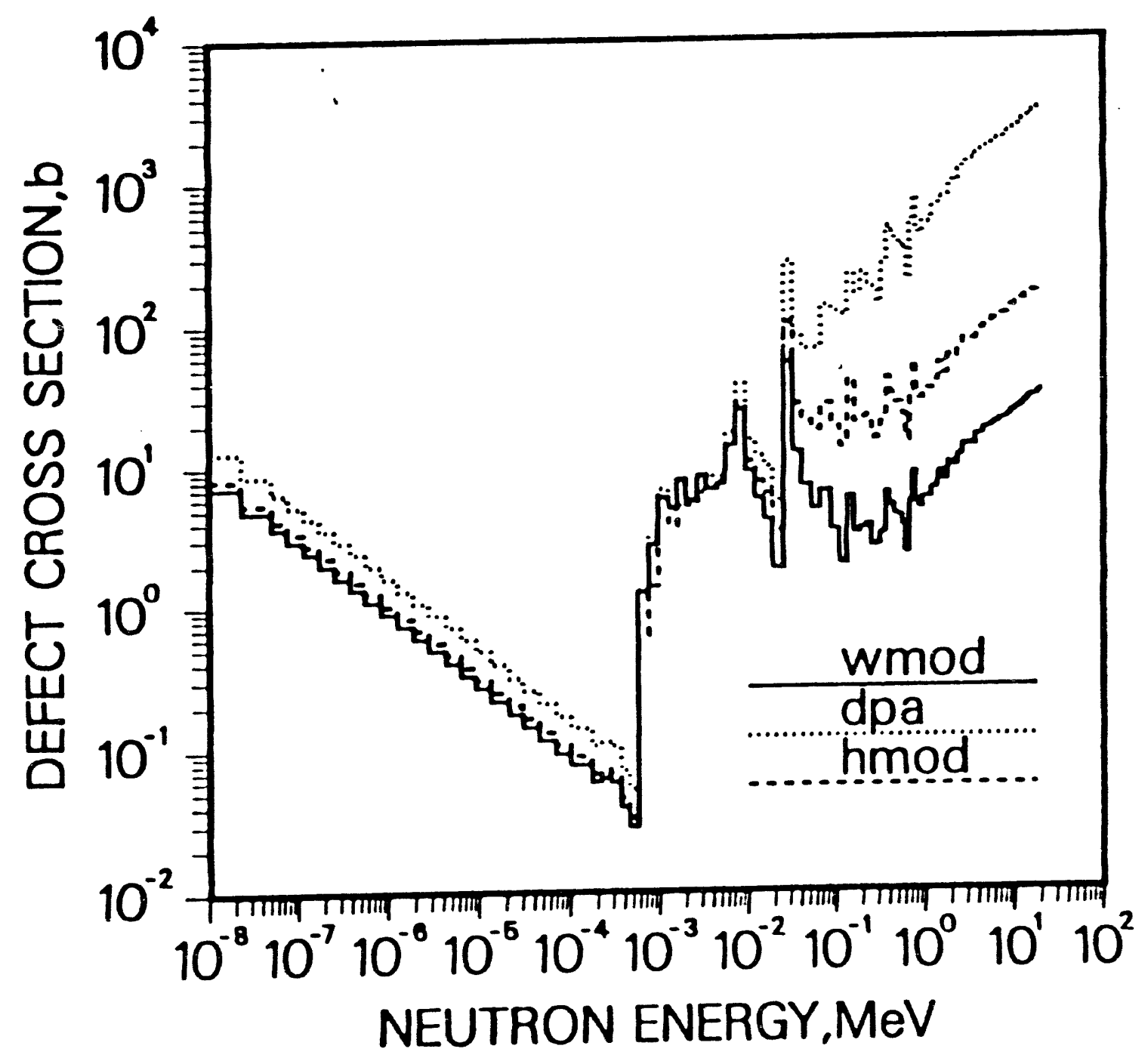

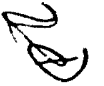




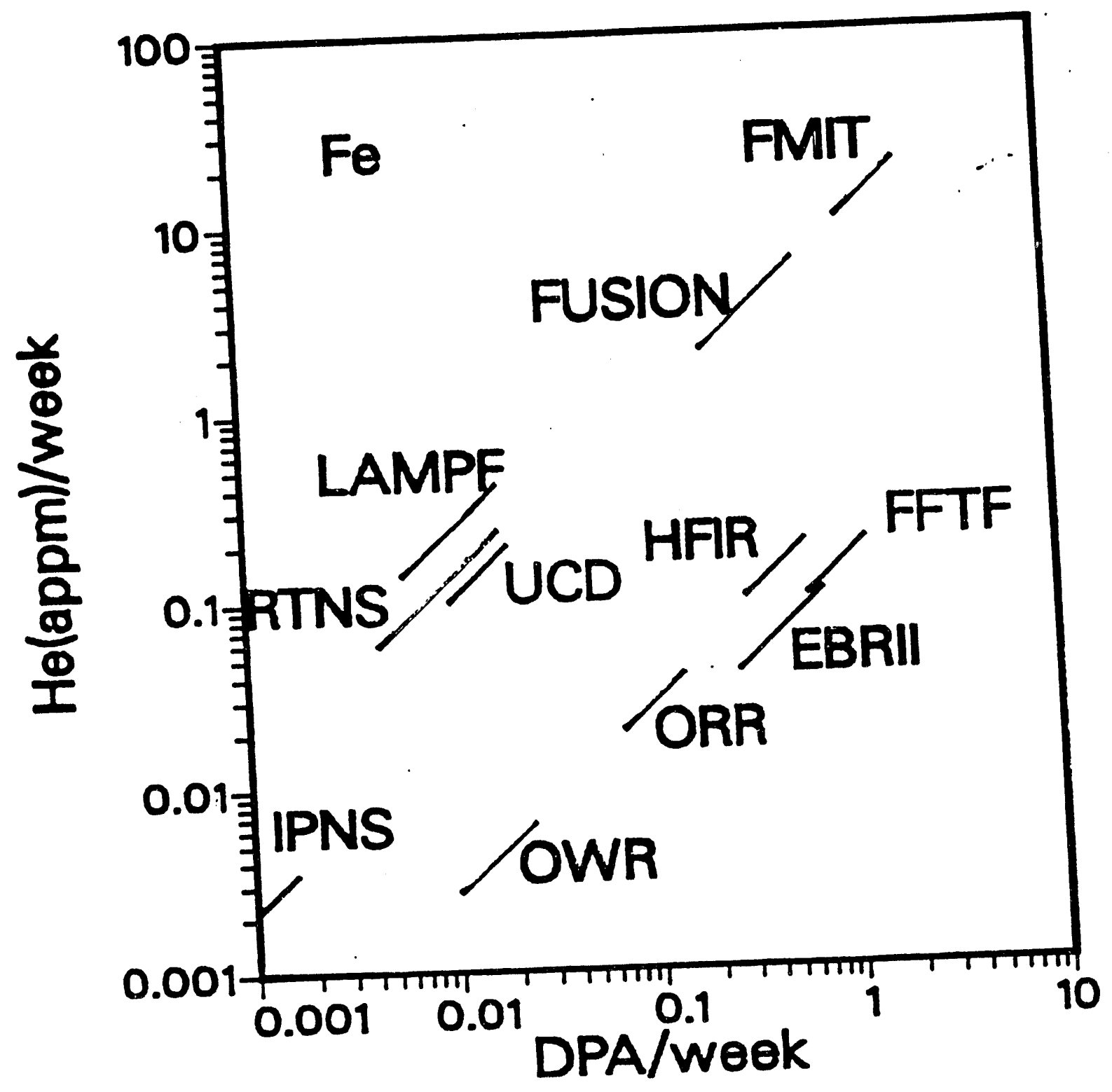



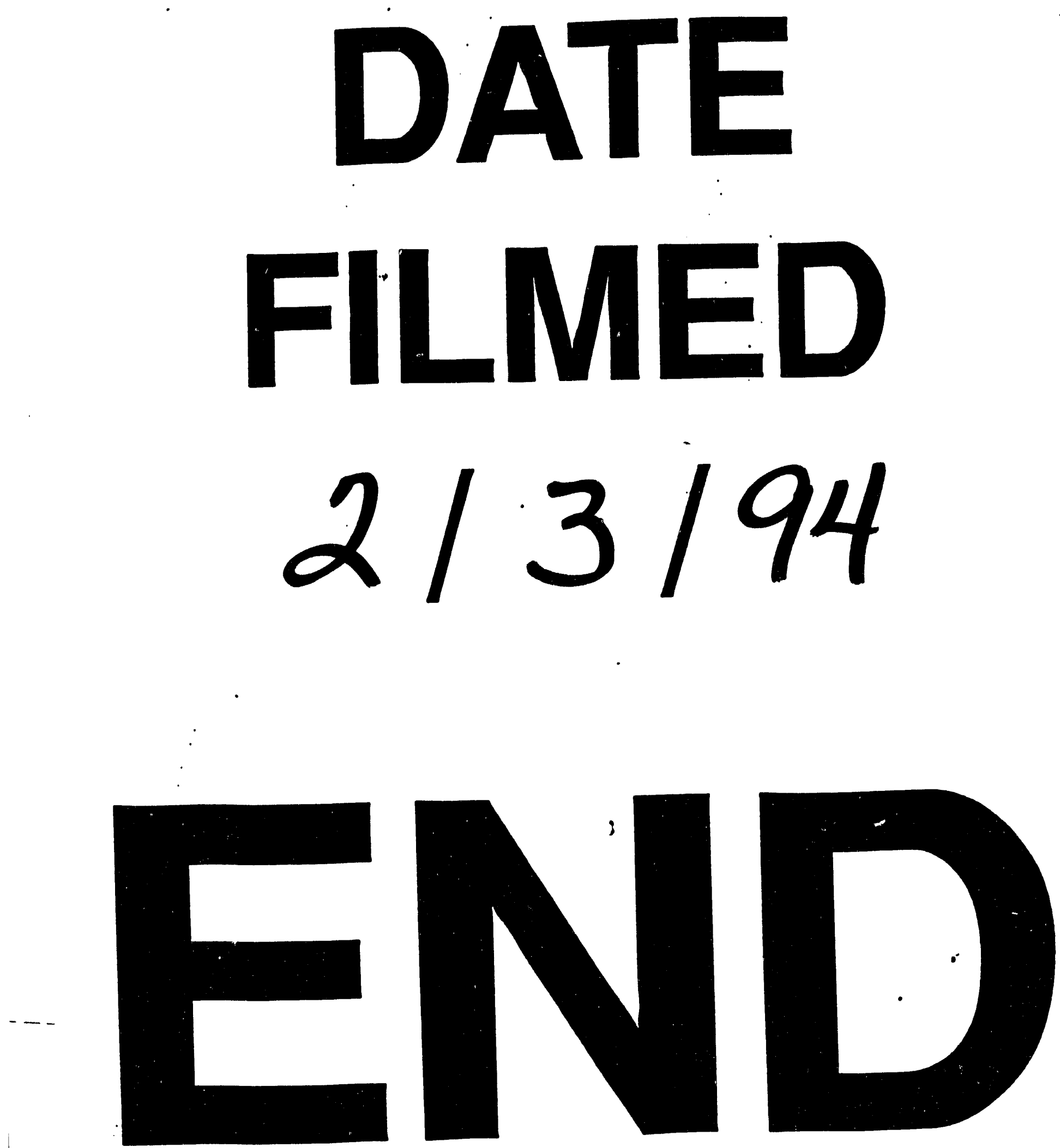
